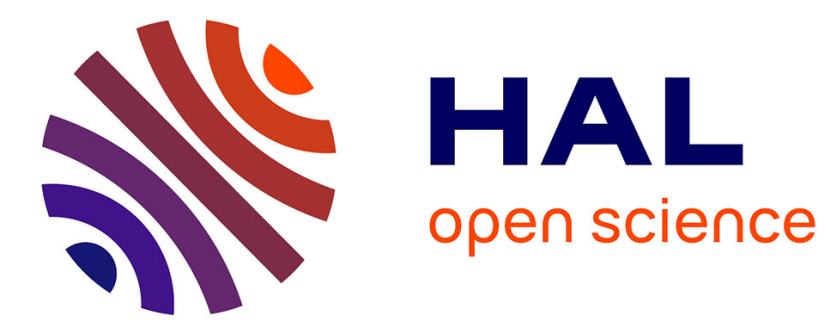

\title{
Du mot à la chose : le travail (dé)construit par le droit ?
}

Claude Didry

\section{To cite this version:}

Claude Didry. Du mot à la chose : le travail (dé)construit par le droit ?. L'Homme et la Société, 2021, nº 212 (1), pp.127-167. 10.3917/lhs.212.0127 . halshs-03318592

\section{HAL Id: halshs-03318592 \\ https://shs.hal.science/halshs-03318592}

Submitted on 2 Jan 2022

HAL is a multi-disciplinary open access archive for the deposit and dissemination of scientific research documents, whether they are published or not. The documents may come from teaching and research institutions in France or abroad, or from public or private research centers.
L'archive ouverte pluridisciplinaire HAL, est destinée au dépôt et à la diffusion de documents scientifiques de niveau recherche, publiés ou non, émanant des établissements d'enseignement et de recherche français ou étrangers, des laboratoires publics ou privés. 


\title{
Du mot à la chose : le travail (dé)construit par le droit ? Du travail normatif à la normativité du travail
}

\author{
Claude Didry (sociologue, CNRS, Centre Maurice Halbwachs, ENS 48 boulevard \\ Jourdan, 75014 Paris) \\ claude.didry@ens.psl.eu
}

\section{Paru dans L'Homme \& la Société 2020/1 n²12 n $^{\circ}$ pages 127 à 167, consultable sur CAIRN}

\begin{abstract}
Despite recent reforms (2016, 2017), labor law remains the major reference for the French workforce where stable employment predominates. To explain such a situation, the text goes back to the genesis of labor law. He first questions, from a reading of the Capital by Marx, the difficulty of understanding labor for the actors themselves, in a rural society dominated by home production where the reference to piecework contract dominates. Analytical concept of classical economics, labor gradually becomes an operational concept for observing a social reality, before entering the language of Law from the adoption of a labor code. Result of a normative work contributing to its identification by the actors in their social life, labor as the object of the employment contract represents the activity of an individual, the worker, for another, the employer. But the employment contract is also the gateway to a community, the staff of the establishment opening the way to union and elective representation carrying work normativity.

Based on this sociohistorical observation, the text finally envisages the scope of the reforms of 2016 and 2017, less as a flexibilization of employment, than as a strengthening of the employer's organizational power in terms of industrial relations. However, recent conflicts show that work retains a substantial part of its normative power.
\end{abstract}

Key words: Home working, piecework contract, subcontracting, labor code, employment contract, reforms

\section{Résumé}

En dépit des réformes El Khomri et Macron, le droit du travail demeure la référence majeure pour une population active où prédomine l'emploi stable. Pour expliquer une telle situation, le texte revient sur la genèse du droit du travail. Il s'interroge en premier lieu, à partir d'une lecture $d u$ Capital, sur la difficulté de saisir le travail pour les acteurs, dans une société rurale dominée par la production à domicile où domine la référence au louage d'ouvrage. Concept analytique de l'économie classique, le travail devient progressivement un concept opératoire pour observer une réalité sociale, avant d'entrer dans la langue du travail à partir de l'adoption d'un code du travail. Résultat d'un travail normatif contribuant à son identification par les acteurs dans leur vie sociale, le travail comme objet du contrat de travail représente l'activité d'un individu, le travailleur, pour un autre, l'employeur. Mais le contrat de travail constitue également la porte d'entrée dans une collectivité, le personnel de l'établissement en ouvrant la voie à une représentation syndicale et élective porteuse d'une normativité du travail.

Partant de ce constat sociohistorique, le texte envisage finalement la portée des réformes de 2016 et 2017, moins comme une flexibilisation de l'emploi, que comme un renforcement $\mathrm{du}$ pouvoir d'organisation de l'employeur en matière de relations professionnelles. Mais, les conflits récents montrent que le travail conserve une partie substantielle de son pouvoir normatif.

Mots clés : travail à domicile, louage d'ouvrage, marchandage, code du travail, contrat de travail, réformes 
Au-delà des incessantes réformes dont il a fait l'objet en France depuis une vingtaine d'années, avec comme point d'aboutissement la loi Travail de 2016 puis les ordonnances Macron de 2017, le droit du travail constitue la référence majeure pour une population active où prédomine l'emploi salarié stable. Face à un mouvement de réforme au long cours, le salariat - sous la figure de cette permanence de l'emploi stable - apparaît comme un fait social prévalant sur sa remise en cause continue par le législateur. En quoi un retour sur la genèse du droit du travail permet-il de retracer l'émergence de ce fait social et expliquer sa portée actuelle?

Il est courant de voir dans la suppression des corporations par la Révolution française la base d'un libéralisme ouvrant la voie d'une soumission des ouvriers à des patrons et, dans son sillage, permettant une révolution industrielle caractérisée par la mécanisation des activités productives dans de grands établissements. Face à une misère ouvrière présentée comme la source d'une «question sociale», le droit du travail correspondrait à la reconnaissance d'un rapport inégal entre ouvrier et patron pour mieux l'atténuer par des garanties annonçant la protection sociale de l'après-guerre. Mais la réalité historique apparaît plus complexe, notamment pour le cas de la France marquée par une diffusion durable de l'activité artisanale à domicile - tant dans les villes que dans les campagnes - que conforte la Révolution (Noiriel, 1986, 2018. Les activités productives y sont placées sous le sceau de l'indépendance revendiquée par les ouvriers pendant la Révolution, que consacre la reconnaissance du louage d'ouvrage par le Code civil en $1804^{1}$, contre la discipline d'un régime corporatif discrédité par son souci d'asseoir la subordination des ouvriers à leurs maitres (Cottereau, 2002). Ainsi, il y a lieu de s'étonner que le droit du travail établi au Xx siècle par l'adoption d'un code du travail consacre un contrat de travail caractérisé, dans la jurisprudence de la Cour de cassation, par le critère de la subordination juridique.

Notre hypothèse est que le droit du travail structure une «vision juridique du monde », rendue possible par «la présence de règles de droit qui la construisent en fixant les rôles d'employeur et de salarié. » (Jeammaud, Le Friant \& Lyon-Caen, 1998 : 359). En effet, cette «vision juridique du monde » oblige à sortir d'une assimilation des activités productives à une «prestation de service»-nom moderne du louage d'ouvrage (article $1710 \mathrm{du}$ Code civil) - répondant à la commande d'un client relayée par un négociant, pour partir du travail comme activité d'une personne - le travailleur - destinée à une autre personne - l'employeur. Cela implique d'envisager ce processus de reconnaissance du travail salarié, comme une «conversion $»^{2}$ de la «vision juridique » projetée par le droit sur les activités productives. Dans ce cadre, le droit du travail correspond d'abord à la reconnaissance d'une catégorie juridique nouvelle, le travail, avant que ne se pose la question de l'identification d'un contrat de travail à partir du critère de la subordination juridique ${ }^{3}$. Cela se comprend aujourd'hui à

${ }^{1}$. Article 1710 et articles 1779 à 1799 (Code civil).

2. Le terme de «conversion » nous a été inspiré par Pierre Bourdieu, dans sa sociologie de l'Algérie en guerre, que l'on retrouve dans son dernier ouvrage : «L'observation des conversions forcées, souvent très coûteuses et très douloureuses, que les nouveaux venus à l'économie proprement économique doivent opérer sous la pression de la nécessité permet sans doute de se faire une idée approchée de ce qui s'est passé aux origines du capitalisme, où les dispositions s'inventèrent en même temps que s'instituait peu à peu le champ dans lequel elles s'accomplissaient. » (Bourdieu, 2000 : 16-17).

${ }^{3}$. Pour Supiot (2011 [1994]), le contrat de travail tient à la transaction entre mise à disposition de son corps par le travailleur et rémunération de l'employeur, en suggérant que la subordination se rapproche d'une soumission personnelle associée au travail lui-même. Pour Cottereau (2002), le droit du travail correspond à l'avènement du contrat de travail rabattu sur le louage des domestiques et des ouvriers (article 1780 du Code civil), suggérant une subordination restaurant la domesticité que la Révolution avait écartée et que le Code civil avait encadré limitativement en le réduisant à un aspect du louage d'ouvrage. En fait, la consécration du critère de subordination juridique (contre celui de la «dépendance juridique » défendu dans le sillage du projet de 1906, voir infra) remonte à l'arrêt Bardou rendu par la Cour de cassation en 1931, qui parle de «lien de 
travers le fait que la subordination juridique entre dans un faisceau d'indices, parmi lesquels prime l'existence d'une prestation personnelle de travail. Cela se comprend historiquement, en revenant à l'adoption d'un code du travail à partir de 1910, avant que la Cour de cassation ne consacre en 1931 le critère de la subordination juridique - contre celui de la « dépendance économique » privilégié par le législateur - pour établir l'existence d'un contrat de travail.

Sur cette base et en partant du constat d'une permanence de l'emploi stable, il convient de sortir des motivations officielles qui ont présidé à leur adoption pour voir en quoi les réformes récentes visent moins une flexibilité de l'emploi qu'un renforcement du pouvoir de l'employeur sur l'organisation du travail et de la négociation collective en privilégiant pour cela le niveau de l'entreprise sur celui de la branche.

Après être revenu sur l'affirmation théorique du travail comme fondement de la valeur d'échange et pierre angulaire de (contre) sociétés utopiques, nous envisagerons la manière dont le droit du travail se dégage contre le louage d'ouvrage. Cela nous permettra d'évaluer la portée des réformes actuelles qui, sans véritablement remettre en cause le droit du travail, portent atteinte à la capacité des travailleurs à participer au pouvoir économique.

\section{La découverte du travail dans une société marchande}

L'historiographie des années 1980 montre, pour un pays comme la France, l'importance d'une activité productive qui, à l'image de la soierie lyonnaise, se diffuse des villes vers les campagnes lyonnaises pendant une grande partie du XIX ${ }^{\mathrm{e}}$ siècle (Noiriel, $1986 \mathrm{ou}$, pour l'Italie du Nord, Dewerpe, 1985). Loin d'une révolution industrielle qui assignerait l'ouvrier à la machine capitaliste, c'est bien plutôt à un développement continu de la production marchande que l'on assiste (Woronoff, 1994), obligeant à s'interroger sur l'existence d'un salariat au sens où nous l'entendons aujourd'hui. Le marxisme courant, reposant sur la détermination « en dernière instance » par les infrastructures techno-économique, semble mis en échec. Mais la lecture du Capital se révèle singulièrement éclairante, en montrant les nombreux obstacles que rencontrent les acteurs économiques -et, en premier lieu, les ouvriers -, dans l'identification du travail que Marx prend comme catégorie cardinale pour dévoiler les mécanismes de l'exploitation. En effet, écartant une évolution unilinéaire menant à la domination exclusive de la «grande machinerie» qui caractérise la fabrique, Marx met également au jour l'existence d'une manufacture et d'un travail à domicile "modernes » en dessinant un univers productif très hétérogène dans lequel l'artisanat est loin d'avoir dit son dernier mot. Dans ces conditions, si Marx parle de travail abstrait, ce n'est pas d'abord, me semble-t-il, comme réduction du travail à une quantité indépendante de sa qualité - la durée du travail qui ne s'impose que progressivement -, par rapport à un travail concret recueillant les savoir-faire du métier, mais comme abstraction d'un travail ramené à une «objectivité fantomatique », qui correspond finalement à son occultation ${ }^{4}$. Côté ouvrier, cette occultation renvoie à une «division naturelle du travail » assignant les membres de la famille à des fonctions naturalisées. Côté capitaliste, elle tient à l'assimilation du profit à un bénéfice commercial. Cela conduit finalement à s'interroger sur la genèse de cette catégorie de travail à

subordination » (Jeammaud, 2020 : 203), revenant sur le critère d'identification du contrat de travail à propos des travailleurs de plateforme).

4. Voir par exemple ce passage du premier livre du Capital : «Considérons maintenant ce résidu des produits du travail. Il n'en subsiste rien d'autre que cette même objectivité fantomatique, qu'une simple gelée de travail humain indifférencié, c-à-d. de dépense de force de travail humaine, indifférente à la forme dans laquelle elle est dépensée. Tout ce qui est encore visible dans ces choses, c'est que pour les produire on a dépensé de la force de travail humaine, accumulé du travail humain. C'est en tant que cristallisations de cette substance sociale, qui leur est commune, qu'elles sont des valeurs : des valeurs marchandes.» (Marx, 1993 [1867] : 43). 
partir de l'usage qu'en fait l'économie classique en prenant le travail comme base de la valeur d'échange, avant que ne s'opère sa révélation aux yeux des acteurs eux-mêmes par la législation (Factory Acts en Angleterre et, oserais-je ajouter, droit du travail en France).

\section{Le travail enfoui sous la division naturelle du travail}

Le travail est couramment associé aux activités matérielles par lesquelles l'Homme produit la réponse à ses besoins, ce qui représente un point de départ dans un grand texte du Capital :

Le travail est d'abord un procès qui se passe entre l'homme et la nature, un procès dans lequel l'homme règle et contrôle son métabolisme avec la nature par la médiation de sa propre action. Il se présente face à la matière naturelle comme une puissance naturelle lui-même. Il met en mouvement les forces naturelles de sa personne physique, ses bras et ses jambes, sa tête et ses mains pour s'approprier la matière naturelle sous une forme utile à sa propre vie. Mais en agissant sur la nature extérieure et en la modifiant par ce mouvement, il modifie aussi sa propre nature. Il développe les potentialités qui y sont en sommeil, et soumet à sa propre gouverne le jeu des forces qu'elle recèle. (Marx, 1993 [1867] : 199)

Mais, ajoute Marx - de manière assez énigmatique :

Nous ne nous occupons pas ici des formes primitives du travail, qui relèvent encore de l'instinct animal. Lorsque le travailleur se présente sur le marché comme vendeur de sa propre force de travail, il a laissé derrière lui dans un passé archaïque l'époque où le travail humain n'avait pas encore dépouillé sa première forme instinctuelle. Nous supposons donc ici le travail sous une forme qui appartient exclusivement à l'homme. (Marx, 1993 [1867] : 200, souligné par l'auteur)

Le capitalisme marqué par la vente de la force de travail, c'est-à-dire la réalisation de produits qui ne satisfont plus immédiatement les besoins du producteur, correspond donc à une rupture dans laquelle le travail perd - nous dit Marx - sa forme instinctuelle ou, pourrait-on penser gagne une dimension consciente. Cela signifie que le travail, avant le capitalisme, conserve une forme instinctuelle. Pour éclairer ce propos, il faut pour moi envisager ce que Marx nomme «la division naturelle du travail » telle qu'elle s'opère dans un cadre familial et principalement en vue de la subsistance de la famille. En effet,

Au sein d'une famille ou, à un degré plus élaboré, d'une tribu, il se crée une division naturelle du travail, à partir des différences de sexe et d'âge, donc sur une base purement physiologique, dont le matériau s'élargit ensuite du fait de 1 'extension de la communauté, de la croissance de la population et surtout des conflits entre différentes tribus et de l'asservissement d'une tribu par une autre. (Marx, 1993 [1867] : 395)

On peut dès lors se demander si la division naturelle du travail dont il s'agit, faisant écho à la forme instinctuelle du travail précédant le capitalisme et la vente de la force du travail repose véritablement sur un travail des individus. Évoquant ainsi le tissage en Inde comme forme emblématique d'une production communautaire, Marx écrit :

C'est seulement l'habileté spécifique accumulée de génération en génération et héritée de père en fils qui confère à l'Hindou comme à l'araignée cette virtuosité. Et cependant, ce tisserand indien exécute un travail très compliqué, par rapport à la majorité des ouvriers de manufacture. (Marx, 1993 [1867] : 383)

Voisinant la production agricole de subsistance, le tissage apparaît comme une forme d'activité qui n'est pas conçue véritablement comme travail par le producteur, mais représente une activité routinière et à certains égards rituelle qui n'est pas sans évoquer, dans sa sociologie de l'Algérie, la situation du paysan ${ }^{5}$ dans la «société traditionnelle » telle que

5. «Le paysan ne se dresse pas comme pouvoir efficace en face d'un monde étranger : très proche d'une nature à peine marquée par l'action de l'homme, il ne peut éprouver que soumission devant des puissances qu'il ne songe pas à discipliner. Faut-il s'étonner qu'il ne saisisse pas son action comme travail au sens vrai, qu'il refuse à 
l'envisage Bourdieu (1977). La production matérielle désigne alors un ensemble d'occupations ou de tâches, prises dans un système d'activités familiales, où domine l'impératif de ne jamais rester inoccupé. Le contrat de travail introduit pour Bourdieu une rupture à certains égards analogue à celle que Marx identifie avec la vente de la force de travail qui extrait le travail de sa forme instinctuelle :

Rien ne s'oppose plus radicalement à l'entraide, qui associe toujours des individus unis par des liens de consanguinité réelle ou fictive, que la coopération qui mobilise des individus sélectionnés en fonction des fins calculées d'une entreprise spécifique : dans un cas, le groupe préexiste et survit à l'accomplissement en commun d'une œuvre commune ; dans l'autre cas, trouvant sa raison d'être hors de lui-même, dans l'objectif futur défini par le contrat, il cesse d'exister en même temps que le contrat qui le fonde. (Bourdieu, $1977: 26$ )

Étant donnée la prédominance des activités à domicile dans la France des XVIII ${ }^{\mathrm{e}}$ et XIX ${ }^{\mathrm{e}}$ siècles, mais aussi la part de la famille et du marchandage dans la production usinière, il me semble alors nécessaire de concevoir un entre-deux entre la forme «instinctuelle » d'une division naturelle du travail qui se maintient et l'éveil d'une conscience individuelle du travail que suscite la participation à une production marchande. Marx le suggère, quand il envisage la mise au travail des femmes et des enfants avec notamment la place des enfants aux côtés des travailleurs adultes dans des industries telles que les installations sidérurgiques.

\section{L'exploitation enfouie sous le commerce, la production sous la circulation}

Ce constat accompagne un autre constat, que l'on peut également tirer $d u$ Capital, c'est celui du salaire à la pièce comme forme «naturelle » de la rémunération de la force de travail dans une société capitaliste, avec pour conséquence le règne d'une multitude d'intermédiaires :

Comme la forme et l'intensité du travail sont ici contrôlées par la forme du salaire elle-même, celle-ci rend superflue une bonne part de la surveillance du travail. C'est pourquoi elle constitue aussi bien la base du travail à domicile moderne précédemment décrit que celle d'un système d'exploitation et d'oppression structuré hiérarchiquement. Ce dernier comprend deux formes fondamentales. D'une part, le salaire aux pièces facilite l'intrusion de parasites entre le capitaliste et l'ouvrier salarié, le sous-affermage du travail [subletting of labour]. Le gain des intermédiaires découle exclusivement de la différence entre le prix du travail que paie le capitaliste et la partie de ce prix qu'il fait parvenir réellement à l'ouvrier. Ce système porte en Angleterre le nom caractéristique de sweating-system. D'autre part, le salaire aux pièces permet au capitaliste de conclure avec l'ouvrier principal - dans la manufacture, avec le chef de groupe, dans les mines avec le haveur, dans la fabrique avec l'ouvrier mécanicien proprement dit - un contrat à tant la pièce, à un prix pour lequel l'ouvrier principal se charge lui-même de recruter et de payer ses auxiliaires. L'exploitation des travailleurs par le capital se réalise ici au moyen de l'exploitation du travailleur par le travailleur. (Marx, 1993 [1867] : 620, souligné par l'auteur).

Cette situation correspond, en France, au cadre juridique dans lequel s'opère en grande partie la production marchande : le louage d'ouvrage. En effet, le louage d'ouvrage est la référence contractuelle qui s'impose le plus souvent pour qualifier les rapports productifs, et, dans le cas de la rémunération à la pièce, il en résulte, selon l'article 1799 du Code civil, que l' "ouvrier est entrepreneur en la partie qu'il traite ». Cela suggère que, dans la production de marchandises, le droit éclaire en priorité la commande qu'un négociant-entrepreneur passe à un ouvrier, laissant à celui-ci toute latitude pour réaliser l'ouvrage, tant par le recours à des membres de sa famille, que par celui à d'autres louages d'ouvrage selon une logique de sous-

traiter comme matière brute cette matière omnipotente que ses croyances peuplent de prestiges et de mystères, qui est le lieu d'un sacré diffus et impersonnel, sources de tous les malheurs et de tous les bienfaits ? Le paysan ne travaille pas à proprement parler, il peine. "Donne à la terre (ta sueur), elle te donnera", dit le proverbe. » (Bourdieu, $1977: 36$ ) 
traitance en cascade pour laquelle on parlera alors de marchandage ${ }^{6}$, dont un décret de la commission du travail dite « du Luxembourg » pris le 2 mars 1848 entend éliminer les formes les plus oppressives car entachées d'une « exploitation de l'ouvrier par l'ouvrier ». Le droit, par le décret interdisant le marchandage, indique l'existence d'une pratique exhumée par Mottez (1966), dont Dewerpe (1989) montre l'extension insoupçonnée jusque-là.

Mais alors, il devient difficile d'identifier une véritable « classe ouvrière » face à celle des «capitalistes », dans la mesure où des intermédiaires ouvriers s'interposent entre les capitalistes qui les ont engagés et les ouvriers qu'à leur tour ils engagent. Ainsi, se pose finalement la question de savoir « où finit l'ouvrier et où commence le patron » (Gaillard, 1997 [1976] ; citée par Noiriel, 2018 : 359). On doit sans doute ici s'en tenir à l'identification de la classe capitaliste, sous la figure de «commerçants »-se distinguant de la masse des citoyens régis par le Code civil par leur assujettissement au code de commerce - dans la mesure où ils ont une pratique habituelle d' "actes de commerce ", c'est-à-dire d'achat pour la revente avec bénéfice selon la formule $A-M-A^{\prime}(A+\Delta A)^{7}$. Cela conduit à un nouvel effacement du travail, cette fois-ci du côté des «capitalistes » convaincus que le bénéfice est moins le fruit d'une «exploitation » de travailleurs, que d'une activité de négoce où la production se disperse en une succession de louages d'ouvrages assimilables à autant d'achats $\left(\mathrm{A}=\mathrm{A}_{1}+\mathrm{A}_{2} \ldots\right)$ avant la vente de la marchandise $(\mathrm{M}-\mathrm{A}=\Delta \mathrm{A})$. Cette conviction trouve son expression idéologique dans cette économie que Marx qualifie de «vulgaire », sous la figure notamment de Frédéric Bastiat, en faisant notamment du libre-échange la source du profit. Ce n'est qu'en ramenant la production marchande au travail objectivé par sa durée, dans le droit chemin de l'économie classique, que Marx peut avancer que le surplus dégagé par la vente de marchandises relève de la survaleur tirée de l'usage de la force de travail par l'entrepreneur capitaliste. Il faut alors attendre la limitation de la durée quotidienne du travail, tout à la fois par les Factory Acts et par les revendications ouvrières, pour que le travail soit saisi par les travailleurs eux-mêmes comme une activité exercée pendant une durée déterminée.

\section{Le travail comme catégorie analytique de l'économie politique}

Ce retour au Marx du Capital permet ainsi de voir à quel point la réalité du travail demeure «fantomatique », alors que le travail hante les théories sociales et les rêves ouvriers du XIX ${ }^{\mathrm{e}}$ siècle, après avoir été pris comme mesure de la valeur d'échange par les économistes classiques dans le siècle précédent. Comme le montre Foucault (1990 [1966]), le travail se présente d'abord comme un concept analytique, au cœur d'une révolution intellectuelle qui se joue au tournant des XVIII ${ }^{\mathrm{e}}$ et $\mathrm{XIX}^{\mathrm{e}}$ siècles, à travers l'économie politique classique. Dans la pensée de Smith, il se présente comme le principe d'une équivalence des marchandises, lié à l'élaboration d'un tableau taxinomique de la richesse des nations. Mais,

6. Le marchandage «suppose l'existence de deux contrats de louage à façon distincts : le premier qui intervient entre le fabricant ou l'entrepreneur général et le marchandeur ; le deuxième, entre le marchandeur et les ouvriers qu'il emploie. [...] Le marchandeur est réputé ouvrier à l'égard de l'entrepreneur général ou du fabricant ; il fait office de maître vis-à-vis de ses propres ouvriers » (Mollot, 1846 : 106).

7. «Acheter pour vendre, ou pour être complet, acheter pour vendre plus cher, A-M-A', semble à vrai dire n'être la forme adéquate que d'une seule espèce de capital, le capital de commerce. Mais le capital industriel aussi est de l'argent qui se transforme en marchandise, puis, par la vente de la marchandise, se retransforme en plus d'argent. Les actes, qui se déroulent en dehors de la sphère de la circulation, par exemple, entre rachat et la vente, ne changent rien à cette forme du mouvement. Enfin la circulation A-M-A' se présente dans le capital porteur d'intérêts de manière abrégée, dans son résultat, sans la médiation, en quelque sorte en style lapidaire : A-A', argent égal à plus d'argent, valeur plus grande qu'elle-même.

A-M-A' est donc en fait la formule générale du capital tel qu'il apparaît, immédiatement, dans la sphère de la circulation. » (Marx, 1993 [1867] : 175) 
À partir de Ricardo, le travail, décalé par rapport à la représentation [de la valeur], et en s'installant dans une région où elle n'a plus prise, s'organise selon une causalité qui lui est propre. La quantité de travail pour la fabrication d'une chose (ou pour sa récolte, ou pour son transport) et déterminant sa valeur dépend des formes de production : selon le degré de division dans le travail, la quantité et la nature des outils, la masse de capital dont dispose l'entrepreneur et celle qu'il a investie dans les installations de son usine, la production sera modifiée; dans certains cas elle sera coûteuse, dans d'autres elle le sera moins. Mais comme dans tous les cas, ce coût (salaires, capital et revenus, profits) est déterminé par du travail déjà accompli et appliqué à cette nouvelle production, on voit naître une grande série linéaire et homogène qui est celle de la production. (Foucault,1990 [1966] : 267, souligné par l'auteur).

Le travail se présente donc avec Ricardo comme la base tectonique d'une historicité, affectant tout à la fois l'homme et la nature. En tant que telle, cette théorisation du travail accompagne selon Foucault une transformation radicale de l'utopie au XIX $\mathrm{X}^{\mathrm{e}}$ siècle, sortant d'une "rêverie des origines », pour concerner «la chute du temps plutôt que son matin » (Ibid. : 274).

À partir de cette théorisation économique, le travail prend une dimension nouvelle dans le cadre de ce socialisme dit «utopique » porté par Owen, Saint-Simon, Fourier ou encore Proudhon (quoique celui-ci paraisse marqué par une nostalgie du métier au regard de l'aliénation industrielle à laquelle il réserve le terme de "salariat »). Il s'inscrit dans le constat d'une anarchie productive, avec pour contrepartie l'enfermement des travailleurs dans une activité répétitive, ennuyeuse et épuisante dans les périodes de «presse », ce que cherche à atténuer, par exemple, Fourier, à travers la recherche d'un «travail attrayant » par un jeu sur l'économie des passions humaines conduisant à imaginer la division du travail en petites séquences variées destinées à éviter la lassitude, voire à susciter un plaisir qui rendrait le repos dominical inutile. Le travail est donc au cœur d'expérimentations organisationnelles, qui - au-delà des échecs connus par les contre-sociétés tentées aux États-Unis dans une inspiration fouriériste ou oweniste - sont menées fréquemment par des patrons comme le premier Robert Owen dans son usine de New-Lanark au début du XIX siècle, à travers une amélioration des logements ouvriers, une réglementation de la vente d'alcool, et l'organisation d'une éducation scolaire des enfants, avant que ce dernier ne s'engage dans la lutte pour une limitation légale de la durée du travail ${ }^{8}$. En France, cette démarche expérimentale prend différentes formes, dont la plus connue est le familistère de Guise établi par Godin à partir de 1859 pour loger les ouvriers de son usine, avant de parvenir à une « coopération intégrale » (Dos Santos, 2015) par transfert du capital aux ouvriers. L'entreprise Godin est alors fréquemment présentée comme une des premières figures de ce travail démocratique analysée par Cukier (2018), à la fois comme reconnaissance du travailleur individuel et de sa famille selon un partage entre travail et hors-travail, et comme institution d'une participation démocratique du travailleur au fonctionnement de l'entreprise.

Ce souci de rationaliser la vie ouvrière, dont témoignent ces réalisations patronales de théories «utopiques » aménagées, semble correspondre à ces utopies ou rêves ouvriers (Rancière, 2012 [1981]), tendant plutôt à mettre l'accent sur le partage entre le temps du travail et le temps familial, au risque de reconduire une vision traditionnelle de la famille assignant la femme à la prise en charge de la famille :

8. «Quand Robert Owen, dix ans à peine après le début de ce siècle, non seulement a défendu d'un point de vue théorique la nécessité d'une limitation de la journée de travail, mais a introduit aussi de fait dans sa fabrique de New-Lanark la journée de 10 heures, on s'est moqué de cette "utopie communiste", de même qu'on se moqua de "son association du travail productif et de l'éducation des enfants", ainsi que des coopératives ouvrières qu'il a le premier mises sur pied. Aujourd'hui la première utopie en question est devenue la Loi sur les fabriques, la deuxième figure officiellement en toutes lettres dans les "Factory Acts", quant à la troisième elle sert déjà à camoufler des manipulations visant à revenir en arrière. » (Marx, 1993 [1867], note 191 : 335). 
La pensée ouvrière désagrège le discours utopique en le partageant. Du projet fouriériste de l'Association industrielle et domestique, elle ne veut retenir que le premier terme, mettant de côté la réorganisation domestique. C'est ainsi qu'en 1848 des ouvriers venus de différents horizons idéologiques - voire de diverses chapelles utopiques - échangent la spécificité de leur projet de réorganisation globale de la société contre une même idéologie organique, celle des délégués à la Commission du Luxembourg : association des producteurs possédant en commun leurs instruments de travail ; organisation coopérative de la consommation; système d'échange par le jeu d'une banque centrale. Utopie ouvrière organique qui, à travers la pensée des Internationaux et le projet de Bourses du Travail se prolongera dans l'idéal de la révolution syndicaliste. Pensée d'une classe qui se résume au fond toujours en un mot : association en 1848 ; syndicat en 1905, quand Pataud et Pouget présentent leur vision d'un monde futur où celui-ci répondra à tous les problèmes et remplira toutes les fonctions : grisaille d'une seule pensée face à l'imprévu des grandes imaginations utopiques. (Rancière, 1975 : 94).

Il y a donc lieu de considérer, me semble-t-il, l'économie politique et le socialisme utopique dans une phénoménologie du travail que l'on retrouve au cœur $d u$ Capital avec une dimension institutionnelle - institutionnaliste ? - permettant de surmonter le vitalisme lié à la lecture néanmoins séminale de Marx que l'on doit à Michel Henry (Sobel, 2009). C'est ici la législation sur la durée du travail qui contribue à distinguer, dans la vie sociale de ceux que l'on dira travailleurs, le temps du travail. Ainsi, on peut penser que le mot est la catégorie qui va permettre d'identifier la chose sur la base de la schématisation juridique que représente la législation sur la durée du travail. Dans ce processus, le décret du 2 mars 1848 revêt une cohérence qui échappe aux acteurs (Jarrige \& Reynaud, 2011), en associant dans un même geste la pénalisation du marchandage et la limitation de la journée de travail à dix heures.

\section{Le travail révélé par son droit}

L'indignation suscitée par la fusillade du $1^{\mathrm{er}}$ mai 1891 à Fourmies, où la troupe tire sur les manifestants en laissant derrière neuf morts et trente-cinq blessés, tient sans doute en partie à la place que le travail commence à prendre dans les institutions républicaines. En effet, dans la France de la Belle Époque, le travail tend à devenir une catégorie opératoire pour concevoir une législation spécifique fondée sur une observation de ses réalités, à partir de la création en février 1891 - l'année de Fourmies - du Conseil supérieur puis en juillet de l'Office du travail. Dans le champ intellectuel, la thèse de Durkheim - De la division du travail social soutenue et publiée en 1893, marque les esprits. L'activité législative s'accélère avec les lois de 1892 sur le travail de nuit des femmes et des enfants, de 1893 sur l'hygiène et la sécurité des travailleurs et de 1898 sur les accidents du travail, qui s'en tiennent cependant aux seuls «établissements industriels » en tenant à l'écart le travail à domicile. L'élection de quaranteneuf députés socialistes à la Chambre en 1893 conforte et intensifie cette orientation, conduisant en 1899 à l'arrivée du socialiste Alexandre Millerand au ministère du commerce et de l'industrie. L'Exposition universelle de 1900 est placée sous le signe du travail, même si la Tour du travail, monument commandé à Rodin pour cette occasion ne sera jamais construite, et accueille un congrès international de juristes qui se conclut par la création de l'Association Internationale pour la Protection Légale des Travailleurs (Herren-Oesch, 1992).

C'est dans ce contexte qu'en 1898 le député Groussier présente une proposition de code du travail qu'il a élaborée au nom du groupe socialiste, avant que ne s'engage sous l'impulsion ministérielle de Millerand une codification des lois ouvrières et de prévoyance sociale. Le rapport de la commission en charge de cette codification va devenir la base d'un projet de code du travail, dont la Chambre adopte finalement le premier livre «des conventions relatives au travail» en 1910. Entre-temps, de 1904 à 1908, une société constituée par les professeurs de droit, la Société d'études législatives, élabore un projet de loi sur le contrat de travail conçu dans l'intention de remplacer le louage d'ouvrage dans le Code civil, mais pouvant s'inscrire dans le code du travail alors en débats. Pour entretenir cet élan 
républicain, un ministère du travail voit le jour en 1906 dans le Gouvernement Clemenceau, dont la charge est confiée au socialiste indépendant René Viviani. Finalement, le travail entre de manière fracassante dans les institutions républicaines en conduisant - au moins de manière sous-jacente - à son explicitation. Ce faisant, la législation inaugure cette "vision juridique » du travail que la pratique de ce droit contribue à ancrer dans les pratiques économiques.

\section{Le travail et le contrat de travail entrent dans la langue du droit}

La reconnaissance d'un droit du travail par un code et, avec elle, l'entrée du contrat de travail dans la langue du droit qui s'opère dans la première décennie $\mathrm{du} \mathrm{XX}^{\mathrm{e}}$ siècle, ébranlent sérieusement le louage d'ouvrage établi un siècle plus tôt par le Code civil. Il faut alors sans doute prendre comme préalable à cette dynamique législative une forme de «travail juridique du négatif » avec la reconnaissance de la prohibition du marchandage établie par le décret du 2 mars 1848, mais dont la légalité avait été atteinte par une remise en cause de ses dispositions en matière de durée du travail. Tout part d'une action en justice engagée en 1896 par deux maçons participant à des constructions s'inscrivant dans le cadre de l'Exposition Universelle, que la faillite du marchandeur les ayant engagés a privés de salaire. Après une première action déclarée irrecevable par le conseil de prud'hommes, c'est dans un cadre pénal que s'engage une seconde action visant à reconnaître la complicité de l'entrepreneur général avec le marchandeur failli, dans un délit de marchandage. Ces deux maçons ont pour avocat René Viviani, alors député socialiste, qui porte leur cause jusque devant la Cour de cassation. Il faudra attendre un arrêt "assemblée plénière » du 31 janvier 1901, pour que l'affaire soit tranchée, après deux renvois devant des cours d'appel qui refusent de s'incliner. Si dans cet arrêt, le délit de marchandage n'est pas reconnu pour les deux maçons, la légalité du décret est établie en réservant le délit de marchandage à des formes avérées d' "exploitation » (Pélissier, Lyon-Caen, Jeammaud \& Dockès, 2008 : 217-225).

Parallèlement aux développements que prend cette affaire, le projet de code du travail que présente Arthur Groussier en 1898 entend englober les dispositions légales existantes en la matière dans un ensemble plus large intégrant de manière systématique les propositions socialistes. L'intention est de montrer que le code du travail s'inscrit dans une dynamique préexistante, au risque de faire prévaloir dans le code une forme de "compilation ». Certains juristes, comme le lyonnais Paul Pic, en feront la base d'une critique à l'égard du code adopté à partir de 1910, au terme notamment des travaux de la commission de codification des lois ouvrières et de prévoyance sociale, dans la mesure où la codification s'était alors opérée à « législation constante » en indiquant les « lacunes » que révèle une organisation systématique des textes. Mais que le code compile ou qu'il innove, le point important est à mes yeux que la codification implique de définir le champ dans lequel la codification opère. Or, sans que le code finalement adopté ne l'explicite, c'est cette détermination du travail qu'introduit le projet Groussier de 1898, comme en témoignent les premiers articles de ce code :

Art. 1. Sont travailleurs les personnes de tout âge et de tout sexe qui louent leur travail ou leurs services aux personnes, aux sociétés, aux communes, aux départements ou à l'État, sous quelques titres que ce soit, dans les exploitations industrielles, commerciales ou agricoles, les administrations ou les établissements publics ou privés.

Art. 2. Sont employeurs : les personnes, les associés en nom collectif, les chefs ou directeurs de société, administrations ou établissements publics ou privés qui occupent des travailleurs.

Art. 3. Le fait qu'une personne consent à fournir son travail ou ses services à une autre personne, moyennant un salaire, constitue un contrat de travail.

Art. 4. Le travail s'entend de toute activité manuelle ou intellectuelle, par laquelle une personne concourt à la production, l'extraction, le façonnage, la transformation, le transport, l'emmagasinement ou la vente de matières ou produits. (Groussier, $1898: 1424$ ). 
Le travail prend une signification très large, dépassant le partage entre manuel et intellectuel, là où l'ouvrage était marqué par un ancrage ouvrier dans un Code civil réservant aux activités intellectuelles des contrats plus «nobles » comme le mandat associé aux professions libérales. Cette ouverture se retrouve pour le contrat de travail, en intégrant l'État, les communes, les administrations et établissements publics dans la liste des employeurs. Il faut attendre la fin des années 1900, pour que le projet d'un statut de la fonction publique commence à être discuté. Enfin, loin du critère de la subordination juridique que retiendra la Cour de cassation en 1931 , le contrat de travail repose sur le «consentement » à fournir un travail à autrui, moyennant un salaire.

Cet attachement au formalisme contractuel reposant sur le consentement des volontés (dont se distingue par exemple le statut ${ }^{9}$ ), se retrouve dans la définition du contrat de travail avancée dans le projet de loi élaborée par la Société d'Études Législatives entre 1904 et 1908 :

\begin{abstract}
Article Premier. Le contrat de travail est le contrat par lequel une personne s'engage à travailler pour une autre qui s'oblige à lui payer un salaire calculé, soit à raison de la durée de son travail, soit à proportion de la quantité ou de la qualité de l'ouvrage accompli, soit d'après toute autre base arrêtée entre l'employeur et l'employé.

Ne sont pas soumis aux dispositions du présent titre les contrats passés par les personnes qui offrent leur travail non à un ou plusieurs employeurs déterminés, mais au public. (Rousseau éd., 1906: 507-508, souligné par l'auteur).
\end{abstract}

Ces articles appartiennent à un projet de loi qui ne sera jamais adopté, mais ils contribueront à alimenter la doctrine juridique lorsque se posera le problème des critères permettant d'identifier l'existence d'un contrat de travail, notamment à l'occasion de la mise en œuvre des réglementations en matière de durée du travail, d'hygiène et de sécurité ou d'accidents du travail. Ils indiquent aussi le changement considérable de perspectives créé par l'adoption d'un code du travail, dans la mesure où ce corpus juridique part du travail comme activité matériellement observable d'une personne en vue de pouvoir déterminer un (ou plusieurs) employeur. Il se fait jour alors une situation de dépendance économique, tenant à la fourniture du salaire par celui qui sera dit «employeur» à celui qui sera dit «travailleur ». Cela n'est pas sans évoquer la figure du prolétaire caractérisé dans Le Manifeste moins par la subordination qu'il subirait dans son travail, que par le fait qu'il en tire sa subsistance ${ }^{10}$.

À cela s'ajoute le souci de substituer le contrat de travail, au louage d'ouvrage et de mettre en échec le marchandage. En effet, ces ouvriers que, même dans les usines, les directeurs d'établissement présentent comme des tâcherons ${ }^{11}$ embauchant à leur tour des aides dont ces directeurs ne connaissent pas nécessairement l'existence, se convertissent en « chefs d'équipes » dont les membres sont liés par un contrat de travail au même employeur. Plus

9. «Le rapport d'emploi liant tout salarié à son employeur est supposé naître d'un contrat. Cette construction n'est pas la seule concevable, même si elle paraît congruente à la "construction juridique" d'une société qui valorise l'égalité civile et valorise l'accord de libres volontés. Ce rapport juridique pourrait procéder d'un acte unilatéral de l'employeur, analogue à la nomination d'un fonctionnaire, ou résulter du seul fait de l'entrée du travailleur dans l'entreprise, c'est-à-dire de la "relation de travail". » (Jeammaud, Le Friant \& Lyon-Caen, 1998 : 361).

10. «On entend par bourgeoisie la classe des capitalistes modernes, propriétaires des moyens de production sociale et qui emploient le travail salarié. On entend par prolétariat la classe des ouvriers salariés modernes qui, privés de leurs propres moyens de production, sont obligés pour subsister, de vendre leur force de travail.» (Note d'Engels pour l'édition anglaise de 1888).

11. «À Fourchambault et à Rive-de-Gier, la fabrication des roues de wagons passait par des marchés de gré à gré entre les entreprises et des tâcherons qui présentaient leurs équipes comme des «coopératives » pour contourner la loi interdisant le marchandage. » (Noiriel, $2018: 363$ ) 
largement, tous les membres d'un même établissement se trouvent ramenés à la condition commune du contrat de travail ouvrant ainsi à chaque travailleur la porte d'une collectivité d'individus aux qualifications différentes mais complémentaires.

\section{Les effets du droit du travail sur l'industrialisation et le travail à domicile}

L'élaboration d'un code du travail et les débats sur le contrat de travail accompagnent en France le développement d'industries très innovantes dans les domaines de l'automobile, de l'aéronautique, de la construction électrique ou de la téléphonie. Le développement de ces secteurs conduit à l'intégration des activités productives dans des établissements de grande taille, comme par exemple Renault à Billancourt, avec une prise en charge de la production automobile de la fonderie jusqu'au véhicule prêt à l'usage (Fridenson, 1972). Il renforce également le besoin d'un enseignement professionnel qui se fonde sur une vision nouvelle du travail allant au-delà de la division traditionnelle des métiers, pour faire face à la communication entre des catégories professionnelles différentes mais complémentaires. Ainsi, un rapport de 1905 souligne :

\footnotetext{
On ne saurait se passer du producteur humain quand il s'agit d'exécuter une pièce qui exige le concours de l'intelligence, de la réflexion et l'application de connaissances scientifiques exactes. Or les industries dans lesquelles les applications scientifiques sont constantes, deviennent de plus en plus nombreuses; certaines sont de création toute récente, l'automobilisme, l'électricité industrielle et domestique, la traction mécanique sous toutes ses formes, etc. Pour exercer ces industries, pour fabriquer, monter, régler ces appareils et ces machines de plus en plus nombreux et variés, même pour préparer le travail aux machines-outils, il faudra des hommes qui soient plus que d'habiles exécutants; ils devront posséder des connaissances précises en mécanique, en dessin, en géométrie, en technologie, en sciences (électricité, physique ou chimie etc.), il faudra que l'ingénieur ou le chef des travaux ait à sa disposition pour réaliser ses conceptions, des ouvriers qui les comprennent et au besoin même soient capables d'apporter à ses plans certaines modifications dictées par la pratique. ${ }^{12}$
}

La guerre joue alors un rôle d'accélérateur dans le développement de ces activités industrielles, en suscitant l'organisation d'une économie de guerre pour faire face à un conflit qui s'avère durable. Un produit a joué un rôle décisif dans l'organisation d'une économie de guerre en France : l'automobile. Charles Gide et William Oualid soulignent ainsi que « le rôle des véhicules automobiles durant les hostilités fut immense : depuis les autobus de Paris réquisitionnés dans la mobilisation jusqu'aux taxis de la Marne, aux tracteurs d'artillerie lourde, aux camions de Verdun et aux chars d'assaut qui décidèrent de la victoire » (Gide \& Oualid, 1931 : 253). L'organisation d'une économie de guerre sous l'impulsion de Millerand (ministre de la guerre) et de Thomas (sous-secrétaire puis secrétaire d'État à l'armement) suscite alors deux expériences notables dans le travail.

La première est celle de la qualification accompagnant la gestion de la main-d'œuvre rapatriée du front, avec comme objectif le retour des ouvriers «spécialistes » de la métallurgie. Après un retour plus large que prévu de soldats dans les usines, la loi Dalbiez du 17 août 1915 vise alors à l'affectation la plus rationnelle possible de la main-d'œuvre, en vue notamment de ne pas dégarnir le font. Elle conduit les contrôleurs de la main-d'œuvre (inspecteurs du travail mobilisés) à identifier trois degrés de qualification, de manière à organiser le retour sur le front des ouvriers substituables par des femmes ou des immigrés. Le premier degré est celui du «spécialiste » appartenant à la profession, le second degré est celui de l'ouvrier "spécialisé » qui a fait preuve de sa productivité depuis son retour du front, le

12. Rapport présenté au nom de la Commission de l'Enseignement Professionnel par MM. Baudrillard et Rocheron ; cité par Didry (2015 : 45). 
troisième degré est celui du manœuvre, ouvrier non spécialisé qui n'a pas sa place dans l'usine et qui doit de ce fait être renvoyé sur le front.

Cette expérience de la qualification est démultipliée par l'industrialisation qui se prolonge, au lendemain de la guerre, et l'idéologie plus générale de la rationalisation qui l'accompagne (Moutet, 1997). Loin de n'y voir qu'une disparition du métier, conduisant à la déqualification de l'ouvrier, Maurice Halbwachs - témoin direct de la rationalisation au cabinet du secrétaire d'État à l'armement pendant le conflit - y voit alors au contraire une exaltation ouvrière qui ouvre aux travailleurs les portes d'une promotion sociale. Pour lui, en effet :

\begin{abstract}
Ainsi, loin d'être dans la société un espace lacunaire où la nature inanimée montre ses entrailles, l'usine avec tout ce qu'elle contient représente un des produits les plus achevés de l'activité commune des hommes, où l'art et la science humaine trouvent leur plus entière expression. C'est un milieu hypersocial au contraire ; si l'ouvrier y sort de lui-même et cesse de s'y considérer comme une personnalité, ce n'est pas pour se confondre avec les choses, c'est parce que la société tire de lui, au moyen de cette organisation, une somme et une qualité d'activité par où il se dépasse, tout de même qu'au sein d'un groupe en proie à une émotion intense, l'individu s'élève à un degré d'exaltation dont il ne se croyait pas capable. L'activité ouvrière serait moins inhumaine que surhumaine: la conscience de l'ouvrier s'étendrait au lieu de se resserrer, et ne perdrait que ses limites individuelles, pour s'élargir et s'approfondir en conscience collective. (Halbwachs, 1920 : 94-95)
\end{abstract}

Il en résulte une capacité de l'ouvrier à partir de son classement dans une catégorie «très étroite » (Halbwachs, $1920: 102)$,

«à s'élever par le passage d'un poste à un autre, d'une usine à une autre car bien souvent sa réflexion se porte [...] sur la technique de son industrie. Et l'on conçoit que s'il a été occupé à plusieurs tâches, s'il a passé par plusieurs usines où tels procédés anciens subsistent, où tels procédés perfectionnés ont été introduits, s'il a par ailleurs quelques connaissances scientifiques, et s'il n'est pas dépourvu d'intelligence, il puisse s'élever à des conceptions voisines de celles sur lesquelles opère l'ingénieur, et même le savant. » (Halbwachs, 1920 : 113).

La seconde expérience tirée de l'organisation d'une économie de guerre est celle de la représentation par des délégués ouvriers appelés à participer à la détermination des salaires dans des commissions régionales mixtes, à partir de 1917, sur la base d'un arrêté d'Albert Thomas. Elle se traduit, à la fin du conflit, par l'émergence d'une revendication nouvelle portée notamment par Léon Jouhaux, le secrétaire de la CGT : celle d'un «contrôle ouvrier » sur l'organisation du travail voire sur la gestion des entreprises ${ }^{13}$, dans le sillage des expériences de participations ouvrières pendant le premier conflit mondial, mais aussi dans celui des conseils ouvriers reconnus par le régime soviétique issu de la révolution de 1917 et la République de Weimar à partir de 1918.

À ces deux expériences tirées des développements intervenus dans le droit du travail au cours de la guerre, il faut ajouter la découverte de la branche une fois la paix revenue. En effet, la mise en ouvre de la journée de huit heures établie par la loi du 23 avril 1919 est renvoyée à des accords d'industrie, regroupant les établissements présentant des caractéristiques techniques similaires. La branche - autre nom de l'industrie - va devenir alors le lieu de classifications professionnelles, encadrant les hiérarchies salariales dans les établissements et reconnaissant la qualification des travailleurs, à partir des conventions négociées en 1936.

Si la mise en œuvre du contrat de travail paraît acquise dans les établissements industriels, le cas des travailleurs à domicile paraît plus problématique malgré l'ambition de

13. Cela que l'on présente alors comme le contrôle ouvrier est envisagé dans l'ouvrage coordonné par François Fagnot (1921). 
les intégrer au salariat dont témoignent les débats de la Société d'étude législative. Les lois de 1915 et 1917 établissant un salaire minimum et la semaine anglaise ${ }^{14}$ pour les couturières à domicile prolongent ces débats, en rattachant celles-ci aux donneurs d'ordre que constituent les grands magasins. Mais la loi du 5 avril 1928 (modifiée par les lois du 5 août 1929 et du 30 avril 1930) sur les assurances sociales suscite un affrontement judiciaire et doctrinal sur l'assujettissement de ces travailleurs, et de leurs employeurs putatifs, à la cotisation sociale. Un rubanier stéphanois est ainsi radié des assujettis à la cotisation sociale, par un jugement du 27 août 1931 rendu par le tribunal civil de Saint-Étienne, à la demande du fabricant pour lequel il travaille, au motif qu'il n'est pas «subordonné» au fabricant en tant qu' «entrepreneur d'ouvrage ${ }^{15}$. La situation sera tranchée par le décret-loi du 28 octobre 1935 ouvrant les assurances sociales aux travailleurs travaillant habituellement pour un ou plusieurs employeurs, en faisant prévaloir le critère de la «dépendance économique » au grand dam de Pic attaché au critère de la subordination ${ }^{16}$.

En 1936, le cas des couturières à domicile se retrouve dans la convention collective « réglant les rapports entre employeurs de la couture et leurs ouvrières dans les départements de Seine et de Seine-et-Oise » signée le 10 juin, deux jours avant la convention des ouvriers de la métallurgie parisienne. La sous-entreprise ouvrière et le travail à domicile sont dans la ligne de mire, mais sans pouvoir arriver à leur suppression pure et simple (Machu, 2011 : 447). Dès lors, le but est de démasquer les «faux artisans », en soumettant cette forme de production aux acquis sociaux du Front Populaire comme la semaine de quarante heures ou les congés payés.

\section{L'affirmation de nouveaux acteurs syndicaux}

Parallèlement à ce développement du droit du travail, le syndicalisme connaît une évolution dans laquelle le travail devient un élément structurant, avec la création d'une Confédération Générale du Travail en 1895. Deux fédérations paraissent alors jouer en son sein un rôle de premier plan, le livre et les chemins de fer. C'est avec le congrès de Montpellier, en 1902, que se dessine une forme nouvelle d'organisation dans laquelle la Confédération articule l'action de fédérations professionnelles nationales à celle d'unions locales. Progressivement, le syndicalisme révolutionnaire, porté par une nouvelle génération, tend à pousser le principe d'une action syndicale distincte de celle des partis politiques, notamment face à la division socialiste et à la méfiance suscitée par la participation ministérielle de Millerand. La grève générale tend à devenir un mythe fondateur, s'incarnant dans la première mobilisation nationale du $1^{\text {er }}$ mai 1906, autour de la revendication de la journée de huit heures. Le congrès d'Amiens, en octobre 1906, établit solennellement la rupture avec le politique, à travers sa fameuse déclaration adoptée à la quasi-unanimité des délégués. Il marque également ses distances avec les projets sur le contrat de travail, présentés comme des « entraves » au développement syndical.

Mais dans cette dynamique, en-deçà de l'ambition de voir le syndicat devenir le nouvel organe central de la société, le travail apparaît comme un point central pour se dégager de la division traditionnelle des métiers - que l'on retrouve en Grande-Bretagne sous la forme des trade unions - et arriver à une restructuration des fédérations de métier en fédérations d'industrie par regroupement de métiers connexes, comme dans le cas notamment de la

14. La semaine dite anglaise correspond à une semaine dans laquelle le samedi après-midi et le dimanche sont chômés. On parle de semaine anglaise, en se référant à une législation britannique de 1854 qui impose le repos du samedi et du dimanche, en rompant avec l'usage du lundi chômé (dit dans la tradition ouvrière « saint lundi »). 15. Recueil Dalloz, 1931, deuxième partie, p. 116.

${ }^{16}$ Voir Pic, 1937. Sur la querelle entre Pic, tenant de la subordination juridique, et Cuche, tenant de la dépendance économique, voir Le Crom (2002). 
fédération du bâtiment. On oublie fréquemment que c'est le Congrès d'Amiens en 1906 qui pose cette orientation, donnant lieu en 1912 à une intervention de Jouhaux sur la recherche de bases rationnelles pour mener à bien cette transformation :

Par le développement du machinisme industriel, l'industrie se trouve être aujourd'hui la réunion de différents métiers, se subdivisant eux-mêmes en différentes spécialités. Ces diverses spécialités sont connexes, soit par l'emploi de la matière, soit par l'objet à confectionner. Comme types de fédérations reposant sur la matière employée, citons les métaux, les cuirs et peaux, etc. Comme types de fédérations reposant sur l'objet à confectionner, citons le bâtiment, le livre, etc. (Jouhaux, 1912 : 53).

Cette orientation se trouve confortée pendant la guerre et au cours de l'après-guerre, avec la loi du 23 avril 1919 invitant à la négociation d'un aménagement de la journée de huit heures au niveau des « industries ».

Parallèlement à la structuration de cette innovation institutionnelle que constitue la CGT, le pluralisme syndical se précise avec la constitution de la Confédération Française des Travailleurs Chrétiens, donnant là aussi la priorité au travail. La création d'une Confédération Générale de la Production Française en 1919 sous l'impulsion d'Étienne Clémentel, ministre $\mathrm{du}$ Commerce et de l'Industrie, esquisse alors un véritable système de relations professionnelles, en établissant les acteurs de négociation tant à l'échelle de l'établissement que de la branche, voire à celle du pays comme ce sera le cas avec l'Accord Matignon signé dans la nuit du 7 au 8 juin 1936.

Dans cette dynamique, la scission de la CGT en 1921 entre une CGT Unitaire (CGTU) proche des communistes et une CGT maintenue conforte cette orientation vers un syndicalisme d'établissement et de branche. En effet, pour la CGT, l'établissement correspond à une base pour établir le contrôle ouvrier dans le cadre de "nationalisations industrialisées»dans des secteurs innovant tel que celui de la «houille blanche». La politique de la CGTU déploie, quant à elle, cette orientation dans son organisation même des « comités d'usine » constitués de représentants élus par les salariés à partir du noyau que constitue la «section syndicale ». Cette forme d'organisation guidée par l'objectif d' "unité d'action à la base » en vue d'arriver à un "front unique de classe », se retrouve tout au long de l'entre-deux-guerres, mais subit des inflexions sensibles dans les années 1930, pour dépasser le sectarisme gauchiste des années 1920 et préparer la réunification de la CGT qui intervient en 1936 dans la dynamique de Rassemblement Populaire contre la montée du fascisme.

\section{Une reconfiguration de l'emploi à partir de la qualification}

L'occupation des usines - qui prend une ampleur inédite au cours du mois de mai 1936 en partant du secteur de l'aéronautique et de l'armement - correspond pour les ouvriers à une véritable exploration des établissements et conduit à l'expérimentation d'une solidarité nouvelle. Les établissements sont occupés et gardés par des piquets de grève, un comité de grève suit et programme les opérations. Les négociations s'engagent d'abord au niveau de l'établissement et aboutissent parfois à des accords. Elles se poursuivent au niveau de la branche, les accords d'établissement se limitant fréquemment à l'engagement pris par la direction de ratifier l'accord de branche. L'établissement apparaît ainsi au cours des grèves du Front populaire comme la base d'une représentation des travailleurs qui se prolonge avec la reconnaissance de délégués du personnel, et que la législation sur les comités d'entreprise, à partir de l'ordonnance de 1945, commence à formaliser au niveau de l'ensemble du personnel.

Mais cet intérêt pour l'entreprise et l'établissement induit une ouverture sur une catégorie plus large, celle de la branche, que l'on trouve dans la loi du 24 juin 1936 sur les conventions collectives susceptibles de faire l'objet d'une procédure d'extension. En ce sens, 
le niveau de la branche devient un levier pour contrecarrer la puissance d'organisation du travail, de détermination des rémunérations que tendent jusqu'alors à monopoliser les directions. Il devient le point d'entrée pour les syndicalistes, dans l'élaboration d'une classification des emplois, à partir de catégories transversales ${ }^{17}$ dépassant la coupure, entretenue par le patronat, entre ouvriers et collaborateurs, comme en témoigne la signature d'une convention des employés, techniciens, agents de maîtrise et ingénieurs dans la métallurgie parisienne en juillet 1936, appelée par la convention ouvrière de juin.

Cette intégration des ingénieurs avec les «employés, techniciens et agents de maitrise » dans la négociation de conventions collectives est constitutive de la formation de cet étrange groupe social des cadres, qui dépasse la profession d'ingénieur en s'inscrivant dans l'organisation du travail au sein de l'établissement (Boltanski, 1982). Elle a pour contrepoint l'intégration de ces ouvriers sans qualité désignés, par exemple, dans l'industrie chimique, comme des ouvriers de fabrique par les organisations de l'Internationale communiste. Selon Roger Pascré, un des négociateurs de la CGT dans la chimie en 1936,

Avant 36, l'ouvrier d'industrie chimique était considéré, à l'échelle internationale, notamment dans l'Internationale des syndicats rouges, comme un "ouvrier de fabrique", c'est-à-dire un ouvrier quelconque, sorte de manœuvre sans qualification, sans rien. L'effort a été fait, un peu en osmose avec les ouvriers d'entretien - l'aile marchante dans l'industrie chimique du point de vue syndical à l'époque puisque les ouvriers d'entretien avaient une certaine qualification, de considérer qu'effectivement les ouvriers dits "de fabrique" avaient également, les uns ou les autres, des qualifications. ${ }^{18}$

Il se dégage finalement des grèves de mai-juin 1936 et de la loi du 24 juin, un formidable mouvement de classification professionnelle au niveau des branches et donc de reconnaissance des qualifications qui se poursuit à la Libération à travers les décrets « ParodiCroizat », du nom des ministres du travail qui se succèdent de 1944 à 1947. Un des enjeux immédiats est alors d'établir dans les entreprises des hiérarchies salariales justifiées, dans une situation de dirigisme économique marqué par un blocage des salaires. Ainsi, Croizat peut annoncer en février 1946 :

\footnotetext{
Sur un rythme record, nous avons peu à peu achevé la remise en ordre des salaires et activé les classifications d'emplois. Cette besogne ingrate a exigé d'innombrables confrontations entre les délégations d'employeurs et de salariés dans mon cabinet. Il s'agissait de mettre tout l'ordre possible dans l'inextricable complexité de la réglementation antérieure, en supprimant ou en atténuant les désharmonies les plus graves ou les injustices les plus flagrantes, car il fallait d'abord courir au plus pressé. C'est ainsi qu'à ce jour, depuis deux mois, cent-quatorze arrêtés portant fixation de salaires et trente-neuf arrêtés portant classification d'emplois ont été établis. Sur ce nombre, soixante-cinq ont été publiés au Journal Officiel. ${ }^{19}$
}

Au-delà de la conjoncture économique marquée par un dirigisme qui se relâchera dans les années 1950, la branche devient ainsi la voie d'entrée dans la vie sociale des grandes catégories d'ouvrier, employé, technicien, agent de maîtrise, ingénieur et cadre, que l'on présentera comme les catégories «Parodi-Croizat». Ces catégories fournissent aussi la trame d'une «nomenclature des catégories socioprofessionnelles» élaborée par l'INSEE. Elles initient alors une transformation dans la manière de caractériser les emplois : le métier (plombier, tailleur...) s'efface derrière la qualification, c'est-à-dire la position dans une hiérarchie de coefficients balisée par les catégories Parodi-Croizat.

17. Ouvriers (manœuvres, ouvriers spécialisés et ouvriers professionnels), Employés, Techniciens, Agents de Maîtrise et Ingénieurs.

18. Entretien avec Roger Pascré réalisé par Claude Didry le 11 février 1992.

19. Discours du 3 février 1946, au congrès de la Métallurgie de la région de Lyon, p. 2 (archives de la Fédération CGT des Métaux). 


\section{Le désarmement du salariat ${ }^{20}$ face à l'instabilité de l'employeur}

Le droit du travail contribue à révéler le travail sous la forme du salariat, à travers la figure dominante du contrat de travail à laquelle se joint, dans l'emploi public, le statut de fonctionnaire. Face à la remise en cause du louage d'ouvrage comme figure de référence des rapports de production marchande reposant sur une garantie d'indépendance de l'ouvrier, le salariat devient le cadre commun d'une activité productive conçue comme un travail inscrit dans l'emploi que définit le contrat pendant le temps qui s'écoule entre sa formation (embauche) et sa résolution (démission, licenciement, retraite) (Friot, 2012). C'est ce cadre que les réformes menées depuis la Seconde Guerre contribuent à définir, puis à aménager.

Toutefois, cette consécration du salariat autour du contrat de travail laisse dans l'ombre l'une des parties au contrat, l'employeur, qui connaît alors d'importantes évolutions. Cela oblige à s'interroger sur les formes multiples de l'employeur, en sortant d'une naturalisation de la confrontation entre salarié et employeur, pour saisir l'historicité de celuici. Il en résulte un éclairage renouvelé sur les deux dernières réformes de grande ampleur de ces dernières années, la loi du 8 août 2016 « relative au travail, à la modernisation du dialogue social et à la sécurisation des parcours professionnels » sous la présidence Hollande, puis, sous la présidence Macron, les ordonnances du 22 septembre 2017.

\section{La consécration du salariat}

Le contrat de travail se présente comme un contrat individuel, liant un travailleur à un employeur. Mais, par là-même,

ce contrat, lorsqu'il doit s'exécuter dans une entreprise, insère le salarié dans une collectivité. C'est en qualité de membre du personnel de l'entreprise qu'il bénéficie de certains droits (participation aux droits dits collectifs, droit d'expression) ou bénéficie de certaines protections (en matière d'hygiène et de sécurité par exemple) [...] C'est dans cette seconde sphère que le rapport de travail s'articule avec les relations professionnelles (Jeammaud, 1989: 301).

Par sa dimension inextricablement individuelle et collective, le contrat de travail se trouve lié à la détermination d'une représentation des travailleurs comme personnel d'une entreprise, circonscrivant la collectivité immédiate de travail dans laquelle s'inscrit le salarié, notamment à partir de l'ordonnance du 22 février 1945 créant les comités d'entreprise.

\section{Acte 1. La sécurité sociale}

De manière plus large encore, la sécurité sociale qui s'organise à partir de la promulgation d'un «plan complet de sécurité sociale » par l'ordonnance du 4 octobre 1945 contribue, par l'institution d'un régime général, à la reconnaissance d'une collectivité nationale des travailleurs salariés à laquelle se joignent les fonctionnaires pour l'assurance maladie. Élaboré et mis en œuvre de 1945 à 1947 par les ministres Alexandre Parodi et Ambroise Croizat, sous l'égide du directeur de la sécurité sociale, Pierre Laroque. Si la sécurité sociale apparaît comme une «révolution ${ }^{21}$, cela tient au revenu de remplacement qu'elle garantit dans le

\section{Titre inspiré de Meyrat (2018).}

21. «Dans le domaine social, il ne suffit pas d'être un technicien, il faut être un apôtre. [...] La technique, l'organisation ne peuvent être pleinement efficaces que si elles sont animées par une foi profonde, une foi ardente, par cette foi qui a soulevé les fondateurs de la mutualité et du syndicalisme, par la foi dans le progrès social, par la foi de tous ces héros obscurs qui ont fait plus peut-être pour le progrès social et pour le progrès de l'humanité que bien des généraux dont le nom est inscrit en lettres éclatantes dans l'histoire, par cette foi des 
régime général, face aux grands risques sociaux (maladie, accidents du travail et maladies professionnelles, vieillesse). Dans son grand discours du 8 août 1946, Ambroise Croizat souligne la libération qui en résulte pour les travailleurs :

Nul ne saurait ignorer que l'un des facteurs essentiels du problème social en France, comme dans presque tous les pays du monde, se trouve dans ce complexe d'infériorité que crée chez le travailleur le sentiment de son insécurité, l'incertitude du lendemain qui pèse sur tous ceux qui vivent de leur travail.

Le problème qui se pose aujourd'hui aux hommes qui veulent apporter une solution durable au problème social est de faire disparaître cette insécurité. Il est de garantir à tous les éléments de la population qu'en toute circonstance ils jouiront de revenus suffisants pour assurer leur subsistance familiale. C'est ainsi seulement, en libérant les travailleurs de l'obsession permanente de la misère, qu'on permettra à tous les hommes et à toutes les femmes de développer pleinement leurs possibilités, leur personnalité, dans toute la mesure compatible avec le régime social en vigueur. (Croizat, 1946 : 3087 3088).

Dans le même temps, la sécurité sociale introduit une reconnaissance nouvelle du travail, à travers la cotisation sociale unifiée à laquelle se trouvent assujettis les assurés et leurs employeurs. Il en résulte un partage entre le travail déclaré qui donne lieu à cotisation sociale, et le travail dissimulé échappant à celle-ci et tombant dans l'économie informelle. Par son existence même, la sécurité sociale - comme en son temps les assurances sociales, mais avec une intensité bien supérieure - est garante de la couverture sociale des travailleurs et porte ainsi un intérêt à établir l'existence du régime légal qui s'attache à leur travail. Dans ce cadre, les conditions d'immatriculation au régime général que l'on retrouve par exemple dans la loi du 30 octobre 1946 sur les accidents du travail, contribue à associer le travail salarié au critère de la dépendance économique. Mais face à la prégnance du critère de subordination juridique que reconnaît imperturbablement la doctrine juridique en droit du travail, la jurisprudence suscitée par les URSSAF depuis leur création en 1960 traduit un aménagement profond de ce critère auquel la justice se montre particulièrement attachée. En effet, les actions en justice des URSSAF se sont révélées décisives pour tempérer l'assimilation de la subordination juridique à un pouvoir de directive de l'employeur, en y voyant bien plutôt l'appartenance à un «service organisé » sous la responsabilité de celui-ci depuis, notamment, l'arrêt (Assemblée plénière) Hebdo-Presse du 18 juin 1976 de la Cour de cassation.

\section{Acte 2. Le CDI comme «forme normale et générale de la relation de travail »}

Dans ce processus d'institution du travail par le salariat, le contrat de travail lui-même connait des évolutions autour, notamment, du partage introduit par la reconnaissance d'un contrat à durée indéterminée, qui conduit à envisager l'existence de contrat à durée déterminée. Cette reconnaissance part d'une réforme du louage de service (qui renomme ainsi l'article $1780 \mathrm{du}$ Code civil relevant de la catégorie plus générale de louage d'ouvrage) en 1890, qui ouvre à la partie lésée par une rupture brusque du contrat le droit d'agir en justice pour demander des dommages-intérêts. Mais cette ouverture législative est restée lettre morte, dans la mesure où il s'avérait impossible pour le salarié d'établir une intention malveillante de l'employeur dans le caractère brusque de la rupture. Avec la loi du 13 juillet 1973, l'encadrement de la rupture du contrat de travail à durée indéterminée se transforme, en imposant à l'employeur de justifier le licenciement par une cause réelle (établie à partir de faits) et sérieuse (proportionnalité de la décision à l'égard des faits constatés). Il est alors possible au salarié en

Tolain, des Varlin, des Pelloutier, des mutualistes et des syndicalistes, par cette foi qui a été et restera à la base de toutes nos révolutions : car c'est une révolution qu'il faut faire et c'est une révolution que nous ferons.» (Laroque, 2008 : 163) 
CDI touché par un licenciement de saisir la justice, pour constater l'absence de cause réelle et sérieuse ouvrant droit à des dommages-intérêts à hauteur du préjudice subi.

Si le CDI apporte au salarié une garantie sur la continuité de son emploi, résultant de la dissuasion exercée sur l'employeur par la perspective de dommages-intérêts coûteux, c'est aussi dans le souci de susciter un engagement du salarié dans la vie de l'entreprise qu'assume le ministre du Travail de l'époque, Georges Gorse, dans les termes suivants :

Comment veut-on que le salarié accepte de se considérer comme participant et se comporter comme tel si, alors qu'on lui promet un intéressement aux bénéfices et qu'on le fait même accéder au titre d'actionnaire, il a conscience d'être à chaque instant à la merci d'une décision incontrôlée ou incontrôlable ? (Brouté \& Didry, $2006: 59)$.

Comme gaulliste de gauche, le ministre voit ainsi dans la loi, une base pour cette participation que le gaullisme a mis au cœur de sa politique sociale.

Ce faisant, le CDI introduit l'horizon probable d'un emploi stable - du moins en l'absence d'opportunités conduisant le salarié à choisir un emploi plus intéressant en termes de rémunération et de progression de carrière - en étendant, à certains égards, la signification du travail au-delà de la seule prestation fournie dans la durée réglementaire de la semaine, pour envisager une carrière dans l'entreprise. Dans le même temps, il crée une partition dans l'espace des contrats, dont témoignent la régularisation de l'intérim par une loi de 1972 et la loi de 1979 sur le CDD complétée par une ordonnance de 1982 définissant le caractère exceptionnel de ce contrat au regard du CDI. Il en résulte que le CDI se présente comme «la forme normale et générale de la relation de travail » (art. 1221-2 CT al. 1) qui s'impose dans le silence des parties ou en l'absence de justifications permettant le recours à long terme à l'intérim ou au CDD. Il est alors courant de voir dans l'institution du CDI la base d'une « dualisme du marché du travail », en opposant des travailleurs stables bénéficiant par leur appartenance à un «marché interne » d'une espérance de carrière, à des travailleurs précaires rejetés dans le «marché secondaire » (Piore, 1978).

\section{Acte 3. Le travail salarié comme fait social}

Cette théorie de la segmentation, reposant sur l'hypothèse d'une "fuite » de l'employeur devant l'emploi stable, a conduit parfois à voir dans les emplois «atypiques » la base d'un «précariat » (Standing, 2017 [2014]) vu comme une population durablement reléguée à une alternance entre emploi et chômage. Les données de l'Enquête Emploi apportent cependant une réfutation de cette théorie pour la France, en montrant que les contrats «atypiques » touchent en premier lieu les plus jeunes, avant que, l'âge venant, ils n'accèdent à un emploi en CDI (graphique 1). 


\section{Graphique 1. Statuts de la population active en fonction de l'âge en $2018^{22}$}

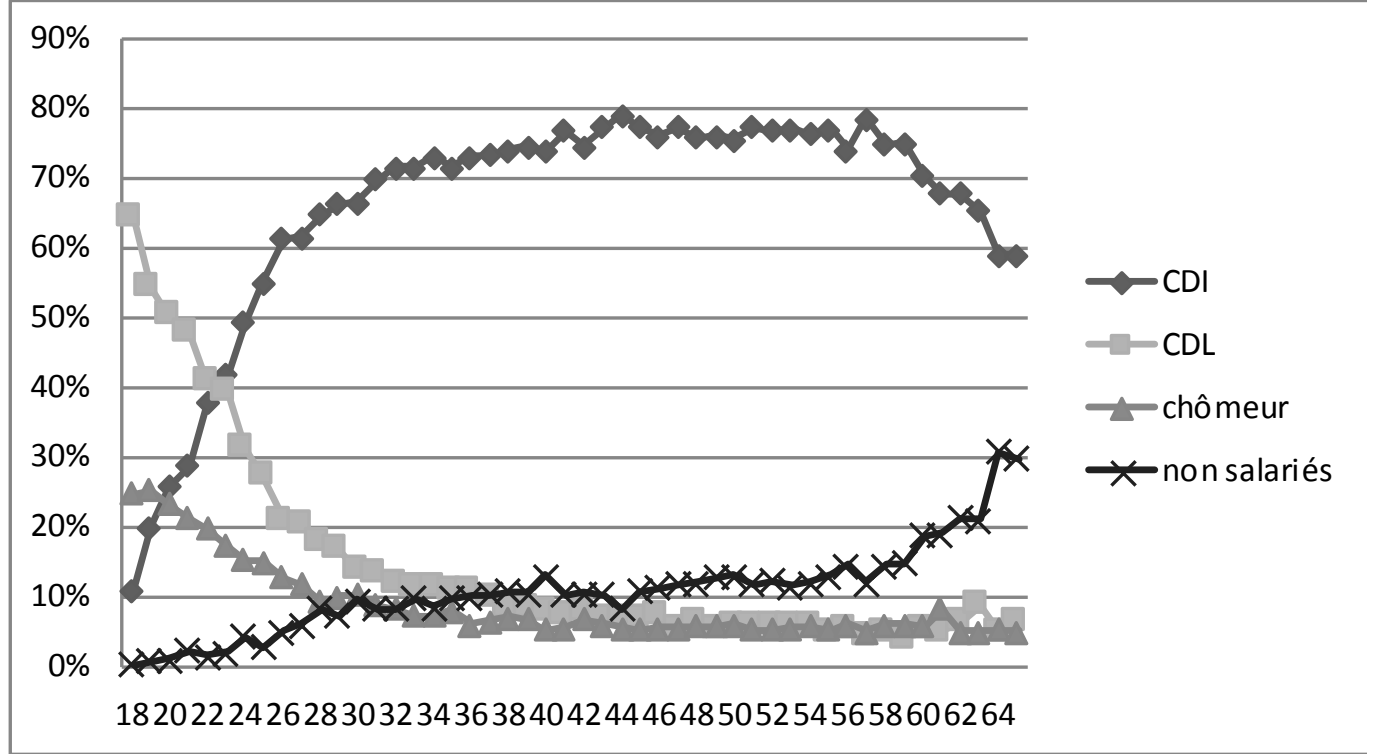

Les emplois en CDI ou en statut de fonctionnaires concernent aujourd'hui environ $70 \%$ de la population active (chômeurs compris). Les emplois « à durée limitée » concernent en priorité les jeunes, qui, cependant, accèdent progressivement à un emploi en CDI ou sous statut entre 18 à 30 ans, forme d'emploi concernant autour de $75 \%$ des classes d'âge entre 30 et 60 ans ${ }^{23}$. Cette place centrale du CDI et de la fonction publique dans la population active s'accompagne d'une stabilité dont témoigne la croissance de l'ancienneté moyenne des salariés dans l'entreprise avec l'âge, culminant à 24 ans pour la classe d'âge 60 ans. Ainsi, l'accès à un emploi stable apparaît comme un horizon raisonnable pour les plus jeunes, avec cependant des trajectoires inégales selon les catégories socioprofessionnelles. Dans le cas des cadres et professions intellectuelles supérieures, la part des emplois en CDL selon l'ancienneté dans l'entreprise pour la population active de la cohorte 1975 (née entre 1973 et 1977) passe ainsi de $30 \%$ à un an d'ancienneté dans la vie active, à $2 \%$ à 20 ans d'ancienneté. En revanche, dans le cas des ouvriers peu qualifiés, elle passe de $40 \%$ à un an d'ancienneté dans la vie active à $25 \%$ à 20 ans (Plault, $2019: 437$ ).

\section{Les dynamiques de l'employeur}

Dans cette percée du salariat reposant sur l'emploi stable en CDI, il reste à éclairer l'autre partie au contrat de travail, l'employeur. En effet, l'employeur ne correspond pas à un «patron » intemporel, qui serait inhérent au capitalisme tel qu'il se développe depuis le XIX siècle. Il constitue une figure juridique liée au contrat de travail, qui s'incarne le plus souvent dans la personne du directeur d'établissement, entité productive coïncidant fréquemment avec le versant économique que constitue l'entreprise dans un capitalisme plutôt familial. Mais cette situation connât une évolution radicale dans les années 1960, sous l'effet d'une politique économique visant à la constitution de «champions nationaux » par intégration des entreprises analogues sur le territoire national. Il en résulte une concentration importante, qui se traduit par la constitution d'entreprises et de groupes de taille nationale, voire internationale, appelés à affronter la concurrence européenne qui s'annonce dans le cadre du

22. Source : Enquête emploi 2017 (mise à disposition par l'ADISP), traitement Marion Plault. Les CDL désignent les contrats à durée limitée (intérim, CDD et apprentissage).

23. Graphique réalisé par Marion Plault à partir des données issues de l'Enquête Emploi de 2018 réalisée par l'INSEE et mise à disposition par l'ADISP. 
Marché Commun. Par exemple, Alsthom prend le contrôle de la société Neyrpic en 1962 dans un processus de regroupement du secteur de la construction électrique, avant que la Compagnie Générale d'Électricité (CGE) ne prenne le contrôle d'Alsthom en 1969. Ces dynamiques capitalistiques s'accompagnent d'une centralisation de la fonction « personnel », visant un contrôle de la «masse salariale » au niveau de l'entreprise, voire du groupe, par une action coordonnée sur les salaires et sur les emplois. Il en résulte de premières « restructurations » destinées à éliminer les «double emploi » par le recours à des départs en préretraite, mais s'accompagnant également d'une hausse continue du chômage qui laisse entrevoir, à la fin des années 1960, la perspective d'un chômage de masse.

Un demi-siècle plus tard, les champions nationaux ont traversé l'épreuve des nationalisations et les grandes réorganisations qui s'en sont suivies, puis celle des privatisations, avec, dans le cas de la CGE, le partage entre Alcatel, absorbé aujourd'hui par Nokia, et Alstom, absorbé aujourd'hui pour partie par General Electrics. Pour saisir la complexité de ce que recoupe aujourd'hui le terme "entreprise ", l'enquête "Liaisons financières » de l'INSEE apporte des éléments importants, en faisant notamment apparaître derrière de nombreuses « unités légales », des «entreprises » construites autour de l'exercice du contrôle financier à partir de « la plus petite combinaison d'unités légales constituant une unité organisationnelle de production de biens et de services et jouissant d'une certaine autonomie de décision » (INSSE, 2018). Pour désigner ces « entreprises » reconstituées par le statisticien, il vaudrait mieux - me semble-t-il - parler de «groupes ». Loin d'une France des PME groupant la majorité des emplois privés, il ressort de cette enquête une concentration productive importante, avec 292 grands groupes de plus de 5000 salariés - mais regroupant près de 30000 unités légales - employant près de 4 millions de salariés du secteur privé, soit près de $30 \%$ de la main-d'œuvre privée. Avec les entreprises de taille intermédiaire de 250 à 5000 salariés, on arrive à plus de 7 millions de salariés, soit plus de la moitié des salariés du privé. Il faut cependant noter le grand écart entre, d'une part, cette concentration économique et financière de la main-d'œuvre et, d'autre part, la dispersion des unités légales. Cette ramification de groupes de taille importante, en une multitude de sociétés, conduit à penser que l'employeur «semble doté d'un étrange pouvoir: il peut se façonner à son gré ; il se donne la forme qui lui convient. Il peut se donner une forme sociétaire, mais il peut, ensuite, se scinder en plusieurs sociétés, se fondre dans une autre, transférer la charge de certains contrats de travail à une autre société... »(Lyon-Caen, 2013).

\section{Des réformes au service de l' "étrange pouvoir » de l'employeur}

Avant de préciser la portée de réformes récentes au regard de cet «étrange pouvoir » de l'employeur, il convient de rappeler les arguments officiels avancés en leur faveur. La loi du 8 août 2016 et les ordonnances du 22 septembre 2017 entendent promouvoir les capacités d'adaptation des entreprises face aux incertitudes que suscite l'entrée dans un "nouveau monde du travail », résultant de la conjonction entre la mondialisation et la croissance du numérique. Elles visent pour cela deux objectifs principaux : l'allègement des risques entourant le licenciement - plus précisément les dommages-intérêts liés à la contestation de la rupture du CDI - et le développement de la négociation d'entreprise destiné à rapprocher la négociation tout à la fois des employeurs et des salariés. Répondant aux besoins des entreprises, elles soulagent également les plus démunis, par une plus grande fluidité/flexibilité de la main-d'œuvre censée faire baisser la part du chômage dans la population active. Comme 
le proclame le propos liminaire d'une tribune d'économistes soutenant la loi El Khomri en mars $2016^{24}$ :

Le chômage, désormais au plus haut niveau depuis l'après-guerre, ne frappe pas tout le monde de la même manière. Il se concentre sur les jeunes et les moins qualifiés. Un chômeur sur quatre a moins de 25 ans, un sur trois n'a aucun diplôme et $80 \%$ n'ont pas dépassé le bac. Ces publics sont les grands perdants d'un marché du travail qui exclut les plus fragiles ou les relègue dans des emplois précaires, tant les entreprises craignent d'embaucher en CDI.

Ces inégalités sont insupportables. En réduisant l'incertitude qui entoure le CDI, le projet de loi El Khomri est de nature à changer la donne : c'est avant tout à ces publics défavorisés qu'elle va donner accès à un emploi durable.

L'ajournement de la barémisation des indemnités par la ministre a sans doute laissé ces économistes sur leur faim, avant que l'ordonnance $n^{\circ} 2017-1387$ du 22 septembre 2017 « relative à la prévisibilité et la sécurisation des relations de travail » ne comble leurs attentes. Mais une relative faiblesse gouvernementale face à un mouvement des chauffeurs routiers, fin septembre 2017, soucieux de maintenir la fixation de leurs «frais de route» par une convention de branche a pu faire craindre que la réforme ne porte pas ses fruits en matière de négociation collective (Cahuc \& Zylberberg, 2018). En effet, grand était le risque de voir la convention de branche reconduite dans ses prérogatives sur des accords d'entreprise privés dès lors de leurs effets d'ajustement au plus près de l'activité économique.

On manque aujourd'hui de recul pour évaluer la portée de la barémisation des dommages-intérêts en cas de licenciement injustifié, mais c'est plutôt sur le terrain de la négociation collective que l'effet de ces réformes est à attendre. Ainsi, par exemple, les accords de performance collective prévus par l'ordonnance $n^{\circ} 2017-1385$ du 22 septembre 2017 «relative au renforcement de la négociation collective » étendent considérablement la capacité de l'employeur à engager des négociations visant à revoir à la baisse les conditions de travail tant en matière de rémunération que de temps de travail, sans que cela n'équivaille à une modification du contrat de travail justifiant un licenciement pour motif économique des salariés réfractaires, leur licenciement étant considéré en tant que tel comme justifié. De plus, rapporté à cet «étrange pouvoir » de l'employeur de s'autodéfinir, le renforcement du poids de la négociation d'entreprise, loin de rapprocher la négociation de l'activité économique, entérine la marge dont l'employeur dispose dans son organisation. En effet, fidèle aux ambiguïtés de la législation en la matière, l'ordonnance relative « au renforcement de la négociation collective » dispose que «[1]e terme "conventions d'entreprise" désigne toute convention ou accord conclu soit au niveau de l'entreprise, soit au niveau de l'établissement » (article 1 al. 3 modifiant l'article L 2232-11 CT). Il en résulte que la négociation d'entreprise s'inscrit dans un ordonnancement hiérarchique spécifique, l'accord de groupe prévalant sur l'accord d'entreprise et celui-ci sur l'accord d'établissement indépendamment du principe de faveur. Or, compte tenu de la responsabilité de l'employeur dans l'organisation de négociations annuelles obligatoires (NAO), c'est finalement ce dernier qui se trouve en mesure de déterminer le niveau de la négociation dans un jeu sur les niveaux lui permettant de limiter les capacités syndicales à porter la voix de leurs mandants. On peut ici faire l'hypothèse que plus le niveau de la négociation s'éloigne de l'établissement, moins les représentants syndicaux seront en mesure de porter la parole de salariés dispersés ou encore de mobiliser ces derniers dans un conflit social susceptible de peser sur les stratégies de l'employeur. Finalement, ce que dessinent les réformes récentes est moins une flexibilisation de l'emploi, qu'une remise en cause du travail démocratique (Cukier, 2018) par une altération des capacités d'expression des salariés et d'intervention des organisations syndicales dans les

24. Collectif, «Le projet de loi El Khomri représente une avancée pour les plus fragiles », tribune publiée le 3 mars 2016 dans Le Monde. 
processus de négociation et des institutions représentatives du personnel ${ }^{25}$. Il y a sans doute lieu ici de parler d'un « coup d'État social » comme le fit Jean-Luc Mélenchon en voyant dans les ordonnances du 22 septembre 2017 un « dix-huit brumaire social » marquant de son sceau la présidence Macron. Il faut ajouter que le terrain avait été préparé par les réformes successives de la présidence Hollande, à commencer par la loi du 14 juin 2013 dite «de sécurisation de l'emploi », démantelant le cadre légal des licenciements collectifs et les ressources juridiques qu'il recelait pour les comités d'entreprise. En ce sens, plus profondément que la violence du dix-huit brumaire, c'est la démoralisation sournoise suscitée par une gauche ayant tourné le dos à ses principes qui s'avère le plus cruel dans cette histoire.

Mais, dans un capitalisme vivant de la rente que les employeurs tirent de leur capacité à agir sur les niveaux de négociation en dissimulant le pouvoir de contrôle financier derrière une multitude de sociétés, il demeure des «points critiques» dont peuvent tirer profit les travailleurs par leur action collective. Le désarmement des salariés résultant des réformes qui se sont succédées récemment ne signifie pas leur dénuement absolu, comme le suggère par exemple la lecture d'un ouvrage écrit avant les dernières réformes par Philippe Askenazy (2016). Ainsi, le mouvement des femmes de chambre qui a touché les palaces parisiens en septembre 2013 à la veille de la fashion week, avec le soutien de la CGT et de la CNT, a ouvert la voie à une activité revendicative posant la question du périmètre de l'entreprise (Askenazy, 2016). Cette grève a eu, pour ces salariées, des résultats immédiats sur les salaires (un treizième mois) et sur la reconnaissance de leur participation aux élections professionnelles dans les établissements des entreprises donneuses d'ordres. Plus près de nous, le conflit social des chauffeurs routiers, en octobre 2017, a suscité une réaction gouvernementale rapide dans le souci d'éviter un blocage économique, alors que les ordonnances visaient à réduire leur champ aux seules rémunérations salariales mensuelles. Il y a peu, le grand mouvement engagé contre la retraite «à points » porté par les secteurs stratégiques des transports et des services publics de l'hôpital et de l'école a prouvé l'existence d'une capacité syndicale de mobilisation à une échelle nationale. Sans céder à l'illusion d'une réplique mécanique aux réformes récentes, on peut penser que le travail salarié n'a pas perdu aujourd'hui toute capacité normative en cédant au destin d'une disparition inéluctable de son droit.

Le contrat de travail a consacré une nouvelle lecture de la production marchande en partant du travail entendu comme l'activité d'une personne, le travailleur, destiné à une autre personne, l'employeur. En cela, il rompt avec la «vision juridique du monde » qui émane du louage d'ouvrage, partant de la commande d'un entrepreneur/négociant à un ouvrier prenant fréquemment lui-même la figure d'un entrepreneur engageant à son tour d'autres ouvriers. Le constat d'une prédominance de l'emploi stable atteste de l'ancrage actuel du travail salarié dans la population active, en suggérant l'existence de collectivités de travail à l'échelle de l'établissement. Si la concentration du pouvoir de contrôle capitalistique tend à s'accroître, la dispersion potentielle des entreprises - et sans doute en grande partie des employeurs reconnus par les contrats de travail-place les organisations syndicales devant une contradiction que renforcent les réformes récentes. En effet, tenant leurs mandats et leurs capacités d'action d'un ancrage dans l'établissement, les représentants syndicaux sont pris dans une tension forte entre d'une part le besoin d'appréhender les dimensions comptables d'une activité économique dépassant leur établissement d'origine en les projetant au niveau

25. Le passage du comité d'entreprise au comité social et économique s'est traduit par une chute du nombre d'élus du personnel, et s'est accompagné de la suppression du Comité d'Hygiène, de Sécurité et des Conditions de Travail (CHSCT). 
de l'entreprise, et d'autre part les dimensions actionnariales à travers lesquelles s'exercent le pouvoir de contrôle financier dans les groupes.

En prétendant promouvoir la négociation d'entreprise, les réformes récentes tendent à renforcer les marges de l'employeur pour se redéfinir dans l'espace qui va du groupe à l'établissement, en passant par l'entreprise-société. Face à un accroissement du pouvoir d'organisation de l'employeur tant sur le travail, que sur les conditions mêmes de la négociation collective, il y a lieu de s'interroger sur la possibilité de démocratiser le travail sur la base de ses caractéristiques actuelles. C'est peut-être alors en clarifiant les ambiguïtés $\mathrm{du}$ droit du travail et de la négociation collective à l'égard de l'entreprise et de ses établissements, que pourrait être envisagé le réseau pertinent des établissements pour concevoir une institution représentative du personnel à l'échelle de cette entreprise clarifiée dans son organisation et ses finalités. Mais avant d'en arriver à cette réflexion, la relative stabilité de l'emploi salarié suggère qu'il y a là place pour un redéploiement méthodique de l'action syndicale au niveau de l'établissement, en visant un renforcement de la syndicalisation et l'expression des questions qui s'y posent, tout en cultivant un potentiel d'actions sur les points critiques qui se révèlent dans la vie économique de l'entreprise.

Dans la crise pandémique que nous vivons aujourd'hui, il faut ajouter que, malgré ce désarmement des salariés et de leurs représentants élus et syndicaux par les réformes récentes, le salariat a été la pierre d'angle d'une politique de préservation de l'emploi par le déploiement du télétravail et le recours massif au chômage partiel. Enfin, la mobilisation suscitée par la multiplication des licenciements collectifs que recense le site stoplicenciement.fr, montre que, au lendemain du grand mouvement social provoqué par la réforme des retraites, la flamme du travail démocratique n'est pas morte. Ici est la rose, ici il faut danser ${ }^{26}$.

\section{Références bibliographiques}

ASKenAZY Philippe, 2016. Tous Rentiers ?, Paris, Odile Jacob.

BOLTANSKI Luc, 1982. Les cadres. Formation d'un groupe social, Paris, Éditions de Minuit.

BoURDIEU Pierre, 1977. Algérie 60. Structures économiques et structures temporelles, Paris, Éditions de Minuit.

—, 2000. Les Structures sociales de l'économie, Paris, Éditions du Seuil.

Broute Rémi \& DIDRY Claude, 2006. «L'employeur en question, les enjeux de la subordination pour les rapports de travail dans une société capitaliste », in $\mathrm{H}$. Petit et N. Thèvenot (dir.), Les Nouvelles frontières du travail subordonné, Paris, La découverte, p. 47-70.

CAHUC Pierre \& ZYLberberg André, 2017. "La réforme n'a pas encore eu lieu », Commentaires [En ligne], 2017, 4 (160), p. 813-818. Mis en ligne le 11/12/2017 (consulté le 16/12/2020). URL : https://www.cairn.info/revue-commentaire-2017-4-page-813.htm ; DOI : $10.3917 /$ comm.160.0813

COLLECTIF, «Le projet de loi El Khomri représente une avancée pour les plus fragiles », Le Monde, tribune publiée le 3 mars 2016.

COTTEREAU Alain, 2002. «Droit et bon droit. Un droit des ouvriers instauré, puis évincé par le droit du travail (France, $\mathrm{XIX}^{\mathrm{e}}$ siècle) », Annales. Histoire, Sciences Sociales [En ligne], 57 (6), p. 1521-1561. Consulté le 16/12/2020. URL:

26. Transposition de l'adage «Hic Rhodus, his saltu» commentée en ces termes par Hegel: «Reconnaître la raison comme la rose dans la croix de la souffrance présente et se réjouir d'elle, c'est la vision rationnelle et médiatrice qui réconcilie avec la réalité, c'est elle que procure la philosophie de ceux qui ont senti la nécessité intérieure de concevoir et de conserver la liberté subjective dans ce qui est substantiel, et de ne pas laisser la liberté subjective dans le contingent et le particulier, de la mettre dans ce qui est en soi et pour soi. » (Hegel 1940 [1820], p. 44). 
Croizat Ambroise, 1946. «Discours prononcé devant l'Assemblée Nationale Constituante, au cours de la deuxième séance du 8 août 1946 », Journal Officiel de la République Française. Débats de l'Assemblée Nationale Constituante, p. 3087-3088.

CUKIER Alexis, 2018. Le Travail démocratique, Paris, PUF.

DEWERPE Alain, 1985. L'Industrie aux champs. Essai sur la proto-industrialisation en Italie du Nord (1800-1880), École Française de Rome.

—, 1989. Le monde du travail en France, 1800-1950, Paris, Armand Colin.

DIDRY Claude, 2015. «L'apprentissage à l'épreuve du droit du travail. De la socialisation familiale à la formation professionnelle (1851-1936)», Artefact, 2, p. 39-52.

Dos SANTOS Jessica, 2015. «De la coopération intégrale à la réforme de l'entreprise : l'intéressement salarial au prisme du Familistère de Guise », L'Homme et la Société [En ligne], 1-2 (195-196), p. 73-86. Mis en ligne le 17/05/2015 (consulté le 16/12/2020). URL : https://www.cairn.info/revue-1-homme-et-la-societe-2015-1-page-73.htm ; DOI : 10.3917/lhs.195.0073

FAGNOT François, 1921. La Part du travail dans la gestion des entreprises, Paris, Maisons Félix Alcan \& Guillaumin réunies-Marcel Rivière et $C^{\text {ie }}$.

FouCAUlt Michel, 1990 [1966]. Les Mots et les choses. Une archéologie des sciences humaines, Paris, Gallimard (Tel).

FRIDENSON Patrick, 1972. Histoire des usines Renault. Tome 1: Naissance de la grande entreprise, Paris, Le Seuil.

FRIOT Bernard, 2012. L'Enjeu du Salaire, Paris, Éditions de la Dispute.

GAILlard Jeanne, 1997 [1976]. Paris, la Ville (1852-1870), Paris, L'Harmattan.

GIDE Charles \& OUALID William, 1931. Le Bilan de la guerre pour la France, Paris, PUF.

GROUSSIER Arthur, 1898. «Proposition de loi sur le code du travail », Journal officiel, «Documents parlementaires », annexe 33, 13 juin 1898, p. 1420-1457.

Halbwachs Maurice, 1920. «Matière et société », Revue Philosophique, 90 (2), p. 88-122.

HEGEL Georg Wilhelm Friedrich, 1940 [1820]. Principes de la philosophie du droit, traduit de l'allemand par André Kaan et préfacé par Jean Hyppolite, Paris, Gallimard.

HERREN-OESCH Madeleine, 1992. "La formation d'une politique internationale du travail avant la Première Guerre mondiale », in J. Luciani (dir.), Histoire de l'Office du Travail, p. 409-426.

INSEE, 2018. Les entreprises en France. Édition 2018, INSEE Références [En ligne], p. 66. Mis en ligne le 08/11/2018 (consulté le 04/01/2021). URL: https://www.insee.fr/fr/statistiques/3639594

JARRIGE François et REYNAUD Bénédicte, 2011. « La durée du travail, la norme et ses usages en 1848 », Genèses, 85 (4), p. 70-92.

JEAMMAUD Antoine, 1989. «Les polyvalences du contrat de travail », Les transformations du droit du travail. Études offertes à Gérard Lyon-Caen, Paris, Dalloz.

—, 2020. «Le régime des travailleurs des plateformes, une œuvre tripartite », Le Droit Ouvrier, 861 (4), p. 181-206.

JeAmmaud Antoine, Le Friant Martine \& LyON-CAEN Antoine, 1998. «L'ordonnancement des relations du travail », Recueil Dalloz, 38, p. 359-368.

JouHAUX Léon, 1912. «Rapport sur la délimitation des Fédérations », dans Confédération générale du travail, XVIII congrès national corporatif (XII de la CGT) et $5^{e}$ conférence des bourses du travail, ou Unions de Syndicats [En ligne], Le Havre, Imprimerie de l'Union, p. 52-53. Consulté le 17/12/2020. URL: https://gallica.bnf.fr/ark:/12148/bpt6k110954f/f100.item.texteImage\# 
LAROQUE Pierre, 2008 [1945], «Discours prononcé le 23 mars 1945 à l'École nationale d'organisation économique et sociale à l'occasion de l'inauguration de la section assurances sociales », Revue française des affaires sociales, 2008/1, p. 151-163.

LE CROM Jean-Pierre, 2002. "Retour sur une "vaine querelle" », in J.-P. Chauchard \& A.-C. Hardy-Dubernet (dir.), Les Métamorphoses de la subordination, Paris, La Documentation Française, p. 71-83.

LYON-CAEN Antoine, 2013. «Retrouver l'entreprise ?», Le Droit Ouvrier, 776 (3), p. 196.

MACHU Laure, 2011. Les Conventions collectives du Front populaire. Construction et pratiques du système français de relations professionnelles, thèse de doctorat d'histoire, sous la dir. de C. Omnès, université Paris-Ouest, Nanterre-La Défense.

MARX Karl, 1993 [1867], Le Capital. Critique de l'économie politique, Livre 1, Paris, PUF.

MARX Karl \& ENGELS Friedrich, 1888 [1848] Manifesto of the Communist Party, trad. de l'allemand par S. Moore et F. Engels, Chicago, Charles H. Kerr \& Company.

MEYRAT Isabelle, 2018. «Droit du travail et droits des travailleurs : le grand désarmement », Droit ouvrier, 837 (4), p. 207-2015.

Mollot François-Étienne, 1846. Le Contrat de louage d'ouvrage et d'industrie, expliqué aux ouvriers et à ceux qui les emploient selon les lois, règlements et usages, et la jurisprudence des conseils de prud'hommes, Paris, Napoléon Chaix et Cie éditeurs.

MotTEz Bernard, 1966. Systèmes de salaire et politiques patronales. Essai sur l'évolution des pratiques et des idéologies patronales, Paris, Éditions du CNRS.

MouTET Aimée, 1997. Les Logiques de l'entreprise. La rationalisation dans l'industrie française de l'entre-deux-guerres, Paris, Éditions de l'École des hautes études en sciences sociales.

NOIRIEL Gérard, 1986. Les Ouvriers dans la société française, XIX ${ }^{e}-X X^{e}$ siècle, Paris, Seuil (Point).

—, 2018. Une Histoire populaire de la France, de la guerre de Cent ans à nos jours, Marseille, Agone.

Pelissier Jean, Lyon-CAen Gérard, Jeammaud Antoine \& Dockes Emmanuel, 2008. Les Grands arrêts du droit du travail, Paris, Dalloz.

PIC Paul, 1937. Note sous Civ. 4 mars, 15 juin et 25 nov. 1936, Recueil Dalloz, première partie, p. 89-91.

PIORE Michaël, 1978. «Dualism in the Labor Market », Revue économique [En ligne], 1, p. 26-48. Consulté le 17/12/2020. URL: www.persee.fr/doc/reco_00352764_1978_num_29_1_408371; DOI : $10.3406 /$ reco.1978.408371

Plault Marion, 2019. Métamorphoses et permanences des parcours professionnels en France (1968-2018). Pour une approche cohortale et sexuée des évolutions de l'emploi, Thèse de doctorat de sociologie, sous la dir. de J. Deauvieau, Université Versailles-Saint Quentin.

RANCIERE Jacques, 1975. «Utopistes, bourgeois et prolétaires », L'Homme et la Société [En ligne], 37-38, p. 87-98. Consulté le 17/12/2020. URL : www.persee.fr/doc/homso_00184306_1975_num_37_1_1600; DOI : 10.3406/homso.1975.1600

—, 2012 [1981]. La Nuit des prolétaires. Archives du rêve ouvrier, Paris, Hachette (Pluriel).

RoussEAU Arthur (éd.), 1906. «Le projet de loi du Gouvernement et le projet de la Société d'études législatives », Bulletin de la Société d'études législatives [En ligne], p. 507-529. Mis en ligne le 17/01/2011 (consulté le 04/01/2021). URL: https://gallica.bnf.fr/ark:/12148/bpt6k5767628n/f507.item

SuPIOT Alain, 2011 [1994]. Critique du droit du travail, Paris, PUF (Quadrige).

Sobel Richard, 2009. «Phénoménologie du travail, ontologie de la vie et critique radicale. Réflexions à propos du statut de l'interprétation de Marx par Michel Henry », Cahiers d'économie politique [En ligne], 56 (1), p. 7-40. Mis en ligne le 11/05/2009 (consulté le 
17/12/2020). URL : https://www.cairn.info/revue-cahiers-d-economie-politique-1-2009-1page-7.htm ; DOI : 10.3917/cep.056.0007

Standing Guy, 2017 [2014]. Le Précariat. Les dangers d'une nouvelle classe, Paris, Éditions de l'Opportun.

WoronofF Denis, 1994. Histoire de l'industrie en France. Du XVI siècle à nos jours, Paris, Seuil. 